\title{
A TOPOLOGICAL APPROACH TO STABILITY OF PULSES BIFURCATING FROM AN INCLINATION-FLIP HOMOCLINIC ORBIT*
}

\author{
SHUNSAKU NII ${ }^{\dagger}$
}

\begin{abstract}
The linear stability problem of multiple pulse solutions of a parabolic system is considered. A topological approach is applied to the eigenvalue problem. Pulses which bifurcate from an inclination-flip homoclinic orbit are treated. A relation between distribution of the eigenvalues and geometry of the homoclinic orbits is proven. In the proof of the theorem, a topological constraint for the distribution of the eigenvalues is discovered and made clear.
\end{abstract}

1. Introduction. The subject of this paper is the linearized eigenvalue problem associated with the stability problem of pulse solutions of a parabolic system. When a pulse solution satisfies certain degeneracy conditions, suitable perturbation generates a solution, called $n$-pulse, which is approximately an alignment of $n$ copies of the original pulse solution. In this article, a relation between the linearized eigenvalue problem of the system along these solutions and the geometric structure of the bifurcation shall be investigated.

First, the notion of a pulse solution is defined. Consider the following type of parabolic system:

$$
U_{t}=\mathcal{D} U_{x x}+f(U)
$$

where $t>0, x \in \mathbb{R}, U \in \mathbb{R}^{l}$ and $\mathcal{D}=\operatorname{diag}\left(d_{1}, \cdots, d_{l}\right)$ with $d_{i} \geq 0$. A system of this type is called a system of reaction diffusion equations if $d_{i}>0$ for all $i$, and is a system of nerve axon equations if $d_{1}>0$ and $d_{i}=0$ for $i \geq 2$. Throughout this paper, the $\operatorname{system}(1.1)$ is assumed to have the zero solution $u(x, t) \equiv 0$ as a stable steady state solution.

A pulse solution-or, more generally, a traveling wave solution-is a solution which decays to zero as $x \rightarrow \pm \infty$ and spatially translates as time evolves, preserving its profile. In other words, $\xi=x+c t$ and $(1.1)$ in $(\xi, t)$ coordinates becomes:

$$
U_{t}=\mathcal{D} U_{\xi \xi}-c U_{\xi}+f(U)
$$

Then a pulse solution $U(x, t)$ of $(1.1)$ is a stationary solution $U(x, t)=U(\xi)$ of $(1.2)$ which satisfies $\lim _{\xi \rightarrow \pm \infty} U(\xi)=0$. That is, if the equation:

$$
\mathcal{D} U_{\xi \xi}-c U_{\xi}+f(U)=0
$$

possesses a solution $U(\xi)$ with that condition, then $U(x+c t)$ in $(x, t)$ coordinates becomes a pulse solution of the system (1.1).

The problem above is regarded as the existence problem of a homoclinic solution in the following manner. The second order ordinary differential equation (1.3) is

\footnotetext{
${ }^{*}$ Received Feb 24, 1999; revised Oct 18, 1999.

tDepartment of Mathematics Faculty of Science, Saitama University, 255 Shimo-Okubo, Urawa 338-8570, Japan (snii@rimath.saitama-u.ac.jp).
} 
rewritten as a first order system:

$$
\left\{\begin{aligned}
U_{1}^{\prime} & =V_{1} \\
& \vdots \\
U_{k}^{\prime} & =V_{k} \\
U_{k+1}^{\prime} & =\frac{1}{c} f_{k+1}(U) \\
& \vdots \\
U_{l}^{\prime} & =\frac{1}{c} f_{l}(U) \\
V_{1}^{\prime} & =\frac{1}{d_{1}}\left\{c V_{1}-f_{1}(U)\right\} \\
& \vdots \\
V_{k}^{\prime} & =\frac{1}{d_{k}}\left\{c V_{k}-f_{k}(U)\right\}
\end{aligned} \quad\left({ }^{\prime}=\frac{d}{d \xi}\right)\right.
$$

where $d_{1}, \cdots, d_{k} \neq 0$ and $d_{k+1}, \cdots, d_{n}=0$, and $f(U)=\left(f_{1}(U), \ldots, f_{l}(U)\right)$. This system is simply written as

$$
u^{\prime}=\mathfrak{X}(u)
$$

where $u=\left(u_{1}, \ldots, u_{l+k}\right)=\left(U_{1}, \ldots, U_{l}, V_{1}, \ldots, V_{k}\right)$. Then $u=\left(U_{1}, \ldots, U_{l}, U_{1}^{\prime}, \ldots, U_{k}^{\prime}\right)$ is a homoclinic solution to the origin of (1.4) if and only if $U=\left(U_{1}, \ldots, U_{l}\right)$ is a solution of (1.3) which converges to zero as $\xi \rightarrow \pm \infty$.

If a homoclinic orbit satisfies certain degeneracy conditions, the system (1.4) undergoes bifurcation generating $n$-homoclinic solutions which correspond to $n$-pulse solutions. More precisely, if (1.4) has a homoclinic solution $h_{0}(\xi)$ with certain properties corresponding to a pulse solution $U_{0}(\xi)$, then a system suitably perturbed from (1.4) possesses a homoclinic solution $h_{n}(\xi)$ which is near an alignment of $n$ copies of $h_{0}(\xi)$; that is, the homoclinic orbit is

$$
h_{n}(\xi) \simeq h_{0}(\xi)+h_{0}\left(\xi+\xi_{1}\right)+\cdots+h_{0}\left(\xi+\xi_{n}\right)
$$

with $0 \ll \xi_{1} \ll \cdots \ll \xi_{n}$. This $h_{n}(\xi)$ naturally corresponds to a pulse solution $U_{n}(\xi)$ of a perturbation of (1.1) which is approximated by an alignment of $n$-copies of $U_{0}(\xi)$ :

$$
U_{n}(\xi) \simeq U_{0}(\xi)+U_{0}\left(\xi+\xi_{1}\right)+\cdots+U_{0}\left(\xi+\xi_{n}\right)
$$

There are two cases for this type of bifurcation:

C: the linearization of (1.4) at the origin has complex principal eigenvalues or

$\mathbf{R}$ : it has only real principal eigenvalues.

Study of the first case goes back to Evans, Fenichel and Feroe [7], and of the second case to Yanagida [20]. [20] inspired several authors to do systematic studies of this problem. See [10] and references therein for more details.

The bifurcation structure under consideration in this article is the bifurcation from what is called an inclination-flip homoclinic orbit, which was analyzed by Homburg, Kokubu and Krupa [9]. The system they treated is three-dimensional, and the equilibrium possesses a two-dimensional unstable manifold and a one-dimensional stable manifold. The system (1.4) falls into this category when $l=2, k=1$ and $c<0$ (Evans [5]), although no concrete example having an inclination-flip homoclinic orbit has been studied until now.

The main interest here is in the stability of these pulses as solution of (1.1). Because it is well known that linear stability of a pulse implies non-linear stability 
of it (Evans [4] and [6], Henry [8] e.t.c.), the eigenvalue problem associated with the linearization along each pulse is investigated:

$$
L P:=\mathcal{D} P_{\xi \xi}-c P_{\xi}+D f(U(\xi)) P=\Lambda P
$$

Proving the stability of a pulse amounts to proving that there is some $\beta<0$ so that the essential spectrum of $L$ is contained in the left half plane $\{\lambda \in \mathbb{C} \mid \operatorname{Re}<\beta\}$ and that there is no eigenvalue with non-negative real part except for a simple eigenvalue at the origin $-L$ has zero as an eigenvalue which corresponds to the spatial translation of the pulse. Notice that the first order system corresponding to (1.5) is of the form of linear perturbation to the linearization of (1.4) along the homoclinic orbit:

$$
\left\{\begin{aligned}
P_{1}^{\prime} & =Q_{1} \\
& \vdots \\
P_{k}^{\prime} & =Q_{k} \\
P_{k+1}^{\prime} & =\frac{1}{c} D f_{k+1}(U) P-\frac{1}{c} \Lambda P_{k+1} \\
& \vdots \\
P_{l}^{\prime} & =\frac{1}{c} D f_{l}(U) P-\frac{1}{c} \Lambda P_{l} \\
Q_{1}^{\prime} & =\frac{1}{d_{1}}\left\{c Q_{1}-D f_{1}(U) P\right\}+\frac{1}{d_{1}} \Lambda P_{1} \\
& \vdots \\
Q_{k}^{\prime} & =\frac{1}{d_{k}}\left\{c Q_{k}-D f_{k}(U) P\right\}+\frac{1}{d_{k}} \Lambda P_{k}
\end{aligned}\right.
$$

where $P=\left(P_{1}, \ldots, P_{l}\right)$. This system is also simply written as

$$
v^{\prime}=[D \mathfrak{X}(U(\xi))+\Lambda B] v,
$$

where $v=\left(P_{1}, \ldots, P_{l}, Q_{1}, \ldots, Q_{k}\right)$ and $B=\operatorname{diag}\left(0, \ldots, 0,-\frac{1}{c}, \ldots,-\frac{1}{c}, \frac{1}{d_{1}}, \ldots, \frac{1}{d_{k}}\right)$.

To tackle the the problem, the following topological approach is employed in this paper.

Let us consider the case where $l=1$ and $\mathcal{D}=1$ :

$$
U_{t}=U_{x x}+f(U)
$$

and assume that this equation has a travelling wave $U(\xi)$. Then the linearized eigenvalue problem associated with $U(\xi)$ becomes

$$
\left\{\begin{array}{l}
P^{\prime}=Q \\
Q^{\prime}=c Q-D f(u(\xi)) P+\lambda P
\end{array}\right.
$$

or

$$
p^{\prime}=A(U(\xi) ; \lambda) p
$$

where $p=(P, Q)$ and

$$
A(U(\xi) ; \lambda)=\left(\begin{array}{cc}
0 & 1 \\
\lambda-D f(U(\xi)) & c
\end{array}\right)
$$


If zero is a stable steady state solution and $U(\xi)$ approaches to it as $\xi \rightarrow \pm \infty$ i.e. $\lim _{\xi \rightarrow \pm \infty} U(\xi)=0$, then

$$
A_{0}(\lambda):=A(0, \lambda)=\left(\begin{array}{cc}
0 & 1 \\
\lambda-D f(0) & c
\end{array}\right)
$$

has one stable eigenvalue and one unstable one if $\operatorname{Re} \lambda>\beta$ for some $\beta<0$.

From now on, let us restrict our attention to real eigenvalues, that is, $\beta<\lambda$ is real and (1.8) is regarded as a system on $\mathbb{R}^{2}$. Then (1.8) induces an equation on $\mathbb{R} \mathbb{P}^{1} \cong \mathbb{S}^{1}$ :

$$
\hat{p}^{\prime}=Y(U(\xi), \hat{p} ; \lambda) .
$$

Let $e^{u}$ be an unstable eigenvector associated with the unstable eigenvalue of $A_{0}(\lambda)$ and $e^{s}$ be a stable one. Then (1.9) has a solution $\hat{p}(\xi ; \lambda)$ which satisfies $\lim _{\xi \rightarrow \pm \infty} \hat{p}(\xi ; \lambda)=\hat{e}^{u}$ if $\lambda$ is not an eigenvalue, where $\hat{e}^{u}$ or $\hat{e}^{s}$ are the points on $\mathbb{R} \mathbb{P}^{1}$ corresponding to $e^{u}$ or $e^{s}$.

Now an index which detects real eigenvalues of the eigenvalue problem (1.8) shall be defined. ( $c f .[11])$

Let us define a map $\mathfrak{g}: \mathbb{S}^{1} \cong \partial\left(\left[\lambda_{1}, \lambda_{2}\right] \times[-1,1]\right) \rightarrow \mathbb{R} \mathbb{P}^{1}$ as

$$
\mathfrak{g}(\lambda, \tau)=\left\{\begin{array}{cll}
\hat{e}^{u}(\lambda) & \lambda \in\left[\lambda_{1}, \lambda_{2}\right], & \tau= \pm 1 \\
\hat{p}\left(\log \left(\frac{1+\tau}{1-\tau}\right) ; \lambda_{i}\right) & \lambda=\lambda_{i} \quad(i=1,2), & \tau \in(-1,1)
\end{array}\right.
$$

for $\beta<\lambda_{1}<\lambda_{2}$ which are not eigenvalues. Then $\mathfrak{g}$ is continuous and induces an homomorphism $\mathfrak{g}_{*}: H_{1}\left(\partial\left(\left[\lambda_{1}, \lambda_{2}\right] \times[-1,1]\right)\right) \rightarrow H_{1}\left(\mathbb{R P}^{1}\right)$.

If there is no eigenvalue in the interval $\left[\lambda_{1}, \lambda_{2}\right]$, then the isomorphism $\mathfrak{g}_{*}$ is trivial. This is because in such case $\mathfrak{g}$ can be naturally extended to a map defined on whole $\left[\lambda_{1}, \lambda_{2}\right] \times[-1,1]$ and thus $\mathfrak{g}$ is homotopic to a map into one point. More over if $\mathfrak{g}_{*}(1)=n$ then there are at least $n$ eigenvalues in the interval $\left[\lambda_{1}, \lambda_{2}\right]$. Here $H_{1}\left(\partial\left(\left[\lambda_{1}, \lambda_{2}\right] \times[-1,1]\right)\right)$ and $H_{1}\left(\mathbb{R P}^{1}\right)$ are identified with $\mathbb{Z}$. (See Fig 1.1)

The stability problem of $n$-pulses was first studied in Yanagida and Maginu's pioneering work [21]. They proved stability and instability of 2-pulses which are generated by the bifurcation structure treated in [7]. Later this result was generalized by Alexander and Jones [2] and [3] to more general systems classified under case C, although the results were restricted to 2- and 3-pulses because of methodological reasons.

Another breakthrough was made by Sandstede [16]. (See also [17], [18] and [22]). He extended what is called Lin's method, which was originally developed to study homoclinic and heteroclinic bifurcations [15], to treat the stability problem for traveling waves generated by the bifurcations. This is a purely analytic tool which reduces the eigenvalue problem for the linear operator to that of a matrix, and the information of the system reduces to the sign and the multitude of coefficients. This approach was so successful that the stability problem for all $n$-pulses was solved both for the bifurcation treated in [7] ([16]) and for the case $\mathbf{R}$ with the weakest degeneracy ([14]).

One problem yet remains: the topological constraint of the eigenvalue problem remains unclear even after stability is obtained by Lin's method. In [21], [2] and [3], the geometry of the bifurcation plays an essential role to determine stability of the 

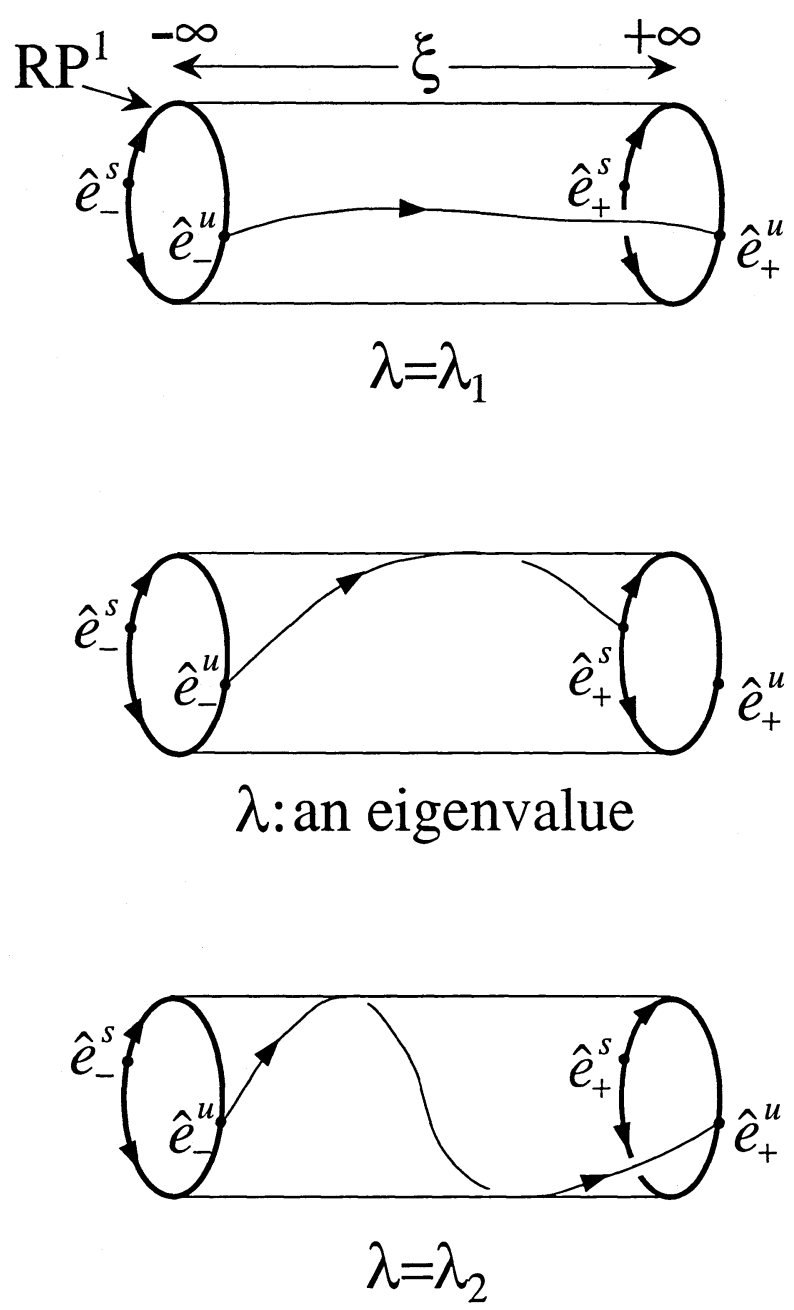

FIG. 1.1.

pulses. At the same time Alexander, Gardner and Jones [1] established a topological invariant in the eigenvalue problem. Later, Nii [13] found another topological constraint, which is similar to what is explained above, in the eigenvalue problem for $N$-front solutions generated by a bifurcation from a doubly twisted heteroclinic loop. This approach contrasts well with Lin's method in [17] which do not tell anything about the topological constraint of the problem.

The purpose of this paper is to reveal that there is a similar topological constraint in the distribution of the eigenvalues associated with the $n$-pulses which is generated by a bifurcation from what is called Inclination-flip homoclinic orbit. Although the result only covers a part of [14], the way in which it is proven provides a good insight into the problem which cannot be obtained from Lin's method.

The following is the main result of this paper-a precise statement of the theorem shall be given in section 3 .

In [9], it was proven that under certain conditions, a suitable perturbation of the 
system creates a suspension of a horseshoe. A periodic symbolic sequence of +1 's and -1 's is assigned to each periodic orbit in this horseshoe. If another perturbation is applied to this system, the periodic orbit disappears in an infinite period bifurcation, generating a homoclinic orbit.

Let $\sigma=\left(\sigma_{0}, \cdots, \sigma_{n-1}\right)^{\infty}$ be such a periodic symbolic sequence, and let $h_{\sigma}(t)$ be an $n$-homoclinic orbit which is generated from the periodic orbit. Then, a number $N(\sigma)$ which is associated with the homoclinic orbit $h_{\sigma}(t)$ is defined as follows.

DEFINITION.

$$
N(\sigma):=\mid 1+\sum_{k=1}^{n-2} \sigma_{1} \cdot \sigma_{2} \cdots \sigma_{k}+\left\{\begin{array}{ccc}
-1 & \text { if } & \sigma_{1} \cdots \sigma_{n-1}=-1 \\
0 & \text { if } & \sigma_{1} \cdots \sigma_{n-1}=+1
\end{array} \mid\right.
$$

Then, the following theorem holds.

THEOREM. The eigenvalue problem (1.6) for $(U, V)=h_{\sigma}$ has at least $N(\sigma)$ negative (positive) eigenvalues if the perturbation is sufficiently small.

This implies that there are stable pulses.

COROLlaRY. In the case of outward twist, the multiple pulse solutions corresponding to symbolic sequences $(-1,+1 \cdots+1$ ) (all symbols are +1 except for the first one) are stable for sufficiently small perturbation if the original 1-pulse is stable.

This paper is organized as follows. In section 2 a review of Homburg, Kokubu and Krupa's result [9] and other necessary information shall be introduced. Section 3 is devoted to a precise statement of the theorem following a geometric interpretation of the problem. Proof of the theorem appears in section 4 and 5; in section 4 a topological index shall be defined, and in section 5 the index is calculated.

2. n-homoclinic bifurcation from an inclination-flip homoclinic orbit. In this section, the result of Homburg, Kokubu and Krupa [9] concerning $n$-homoclinic bifurcation from an inclination-flip homoclinic orbit shall be reviewed.

2.1. Inclination-flip homoclinic orbit. To begin with, the notion of an inclination-flip homoclinic orbit and basic assumptions are introduced.

The subject here is a system of ordinary differential equation defined on $\mathbb{R}^{3}$ :

$$
u^{\prime}=\mathfrak{X}_{0}(u)
$$

satisfying the following conditions.

1. The origin $O$ is a hyperbolic equilibrium of (2.1) and the linearization $D \mathfrak{X}_{0}(O)$ of $\mathfrak{X}_{0}$ around $O$ has real eigenvalues $\lambda^{s}, \lambda^{u}, \lambda^{u u}$ with $\lambda^{s}<0<\lambda^{u}<\lambda^{u u}$.

2. (2.1) has a homoclinic orbit $\Gamma$ to $O$ i.e. $\Gamma=\left\{h_{0}(t) \mid t \in \mathbb{R}\right\}$ and $h_{0}(t)$ is a non-trivial solution of (2.1) which satisfies $\lim _{t \rightarrow \pm \infty} h_{0}(t)=O$.

Condition (1) implies that the system (2.1) possesses an one-dimensional stable manifold $W^{s}(O)$ and a two-dimensional unstable manifold $W^{u}(O)$ at $O$. Furthermore, there exists a two-dimensional invariant manifold $W^{e s}(O)$, called extended stable manifold, the tangent space of which at $O$ is spanned by the eigenvectors associated with eigenvalues $\lambda^{s}$ and $\lambda^{u}$.

The definition of an inclination-flip homoclinic orbit is as follows.

DEFINITION 2.1. A homoclinic orbit $\Gamma$ is called an inclination-flip homoclinic orbit provided that 


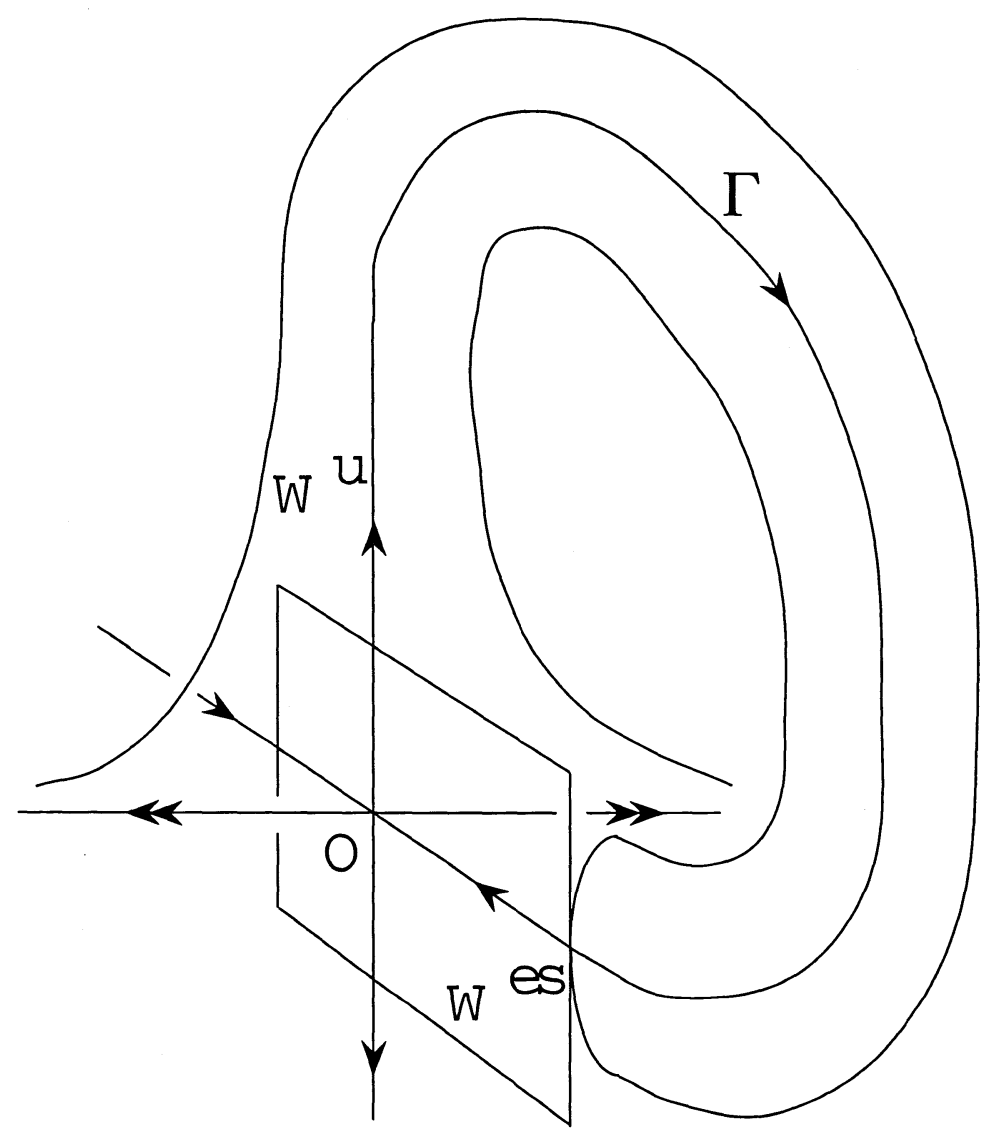

FIG. 2.1.

CT: $W^{u}(O)$ and $W^{e s}(O)$ are tangent along $\Gamma$ (see Fig 2.1),

NR: $\lambda^{u} \neq\left|\lambda^{s}\right|$ and

PR: $0 \neq \lim _{t \rightarrow-\infty}\left|h_{0}(t) e^{-\lambda^{u} t}\right|<+\infty$ and $0 \neq \lim _{t \rightarrow+\infty}\left|h_{0}(t) e^{-\lambda^{s} t}\right|<+\infty$.

In [9], the problem is treated under additional conditions on the eigenvalues and the tangency of $W^{u}(O)$ and $W^{e s}(O)$ :

EV: $\lambda^{u u}>2 \lambda^{u}$ and $-\lambda^{s}>2 \lambda^{u}$;

QT: unstable manifold $W^{u}(O)$ and extended stable manifold $W^{e s}(O)$ have quadratic tangency along homoclinic orbit $\Gamma$.

In order to investigate the bifurcation from an inclination-flip homoclinic orbit, a smooth family of ordinary differential equations on on $\mathbb{R}^{3}$ which unfolds $\mathfrak{X}_{0}$ is considered:

$$
u^{\prime}=\mathfrak{X}_{\mu}(u)
$$

and a return map along the homoclinic orbit and its perturbation by $\mu$ is analyzed.

The return map $f_{\mu}$ is given as the composition of two successive mappings $F$ and $G$ 
between two cross sections $\Sigma_{1}:=\{x=1,|y|,|z|<1\}$ and $\Sigma_{0}:=\{|x|,|y|<1, z=1\}$ :

$$
\begin{array}{rrr}
F: \Sigma_{1} \rightarrow \Sigma_{0}: & (1, y, z) \mapsto & \left(z^{-\frac{\lambda^{s}}{\lambda^{u}}}, y z^{-\frac{\lambda^{u} u}{\lambda^{u}}}, 1\right) \\
G_{\mu}: \Sigma_{0} \rightarrow \Sigma_{1}: & (X, Y, 1) \mapsto & (1, G(X, Y ; \mu)) \\
\text { and } & \\
f_{\mu}: \Sigma_{1} \rightarrow \Sigma_{1}: \quad(y, z) & \mapsto \quad\left(G_{\mu} \circ F\right)(y, z)
\end{array}
$$

Here, the coordinates near origin is denoted by $(x, y, z)$ and the system $(2.2)$ is assumed to be linearized into the following form through a $C^{3}$-coordinate change in a neighborhood $\mathcal{N}$ of the cube $\{|x|,|y|,|z| \leq 1\}$.

$$
\mathfrak{X}_{\mu}=\lambda_{\mu}^{s} x \frac{\partial}{\partial x}+\lambda_{\mu}^{u u} y \frac{\partial}{\partial y}+\lambda_{\mu}^{u} z \frac{\partial}{\partial z}
$$

This linearization is possible under a generic non-resonance condition.

The perturbation parameter is also suitably chosen in the following manner. Corresponding to the existence of the homoclinic orbits $\Gamma$ and the conditions $(\mathbf{C T})$ and (QT), the map $G(X, Y ; \mu)=\left(g^{1}(X, Y ; \mu), g^{2}(X, Y ; \mu)\right)$ satisfies

$$
G(0,0 ; 0)=(0,0), \frac{\partial g^{1}(0,0 ; 0)}{\partial Y}=0, \frac{\partial^{2} g^{1}(0,0 ; 0)}{\partial Y^{2}} \neq 0 .
$$

The second equality together with the fact that $G$ is a diffeomorphism implies

$$
\frac{\partial g^{2}(0,0 ; 0)}{\partial Y} \neq 0
$$

and by implicit function theorem, there exists $Y=Y_{*}(\mu)$ so that $g^{2}\left(0, Y_{*}(\mu) ; \mu\right) \equiv 0$. Then, for a generic two parameter family $\mathfrak{X}_{\mu}$ unfolding $\mathfrak{X}_{0}$, a new parameter $\nu$ can be introduced through the relation

$$
\nu=\left(\nu_{1}, \nu_{2}\right):=\left(g^{1}\left(0, Y^{*}(\mu) ; \mu\right), \frac{g_{Y}^{1}\left(0, Y_{*}(\mu) ; \mu\right)}{g_{Y}^{2}\left(0, Y_{*}(\mu) ; \mu\right)}\right)
$$

where $g_{Y}^{i}$ stands for $\frac{\partial g^{i}}{\partial Y}$.

As the object of interest is the dynamics of the return map, a smaller rectangle $R_{\rho}:=[0, \rho] \times[-1,+1] \subset \Sigma_{0}$, its preimage $C_{\rho}:=F^{-1}\left(R_{\rho}\right) \subset \Sigma_{1}$ under $F$ and its image $P_{\rho}:=G_{\nu}\left(R_{\rho}\right) \subset \Sigma_{1}$ under $G_{\nu}$ is put into focus. For properly chosen $\rho>0$, any orbit which stays in a neighborhood of the heteroclinic orbit $\Gamma$ passes through these sets. Note that $C_{\rho}$ is a cusp-shaped region and the boundary of which consists of two side curves

$$
b_{ \pm}:=\left\{\left( \pm z^{\frac{\lambda^{u u}}{\lambda^{u}}}, z\right) \mid 0 \leq z \leq t_{z}=\rho^{-\frac{\lambda^{u}}{\lambda^{s}}}\right\}
$$

and top segment

$$
t:=\left\{\left(\rho^{-\frac{\lambda^{u u}}{\lambda^{3}}} y, t_{z}\right) \mid-1 \leq y \leq 1\right\}
$$

On the other hand, $P_{\rho}$ is a parabola-like region, and if one of the boundary $p_{0}:=$ $\left\{G_{\nu}(0, Y, 1) \mid-1 \leq Y \leq 1\right\} \subset P_{\rho}$ is expressed as

$$
y=\varphi(z ; \nu)
$$




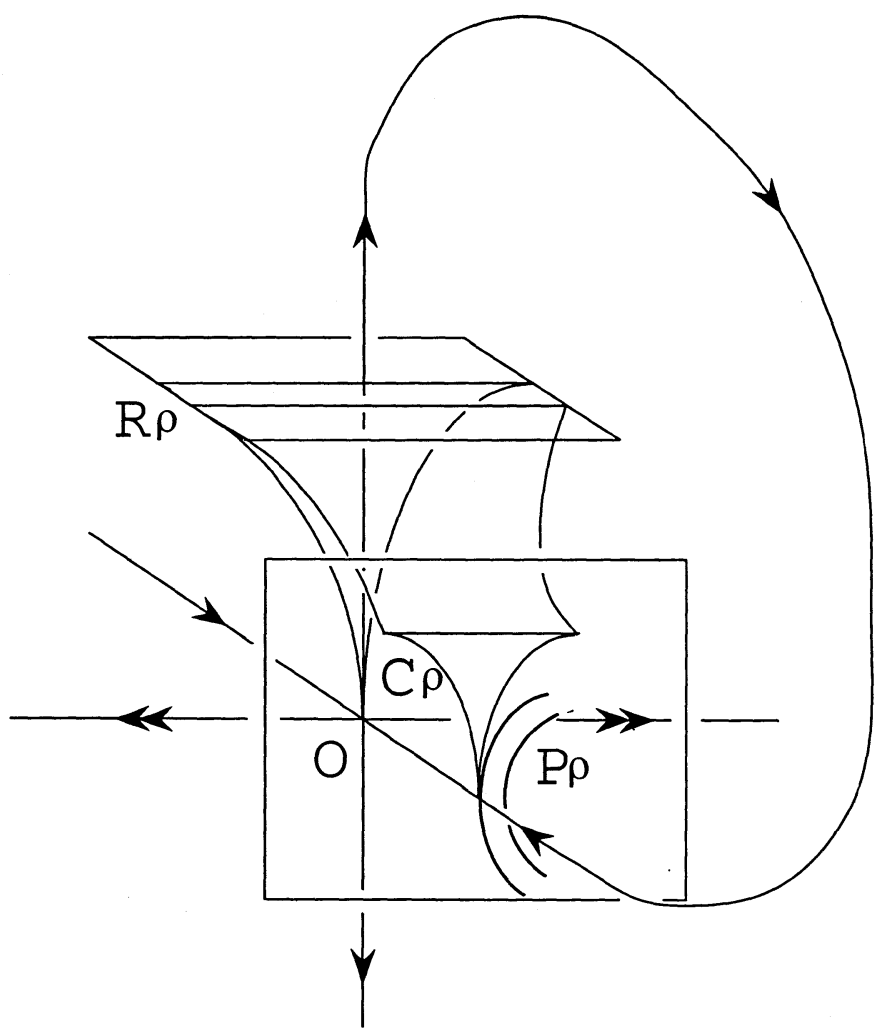

FIG. 2.2.

by eliminating $Y$ from

$$
y=g^{1}(0, Y ; \nu) \quad \text { and } \quad z=g^{2}(0, Y ; \nu)
$$

then

$$
\varphi(0 ; \nu)=\nu_{1} \text { and } \frac{\partial}{\partial z} \varphi(0 ; \nu)=\nu_{2}
$$

hold.

The mutual position of the cusp $C_{\rho}$ and the parabola $P_{\rho}$ at $\nu=0$ is determined by the sign of $g_{X}^{1}(0,0 ; 0) g_{Y Y}^{1}(0,0 ; 0)$, where $g_{X}^{1}$ and $g_{Y Y}^{1}$ stand for $\frac{\partial g^{1}}{\partial X}$ and $\frac{\partial^{2} g^{1}}{\partial Y^{2}}$. In fact, the second derivative of $\varphi(z ; 0)$ at $z=0$ is expressed as

$$
\frac{\partial^{2}}{\partial z^{2}} \varphi(0 ; 0,0)=\frac{g_{Y Y}^{1}(0,0 ; 0)}{\left\{g_{Y}^{2}(0,0 ; 0)\right\}^{2}}
$$

and if $g_{X}^{1}(0,0 ; 0) g_{Y Y}^{1}(0,0 ; 0)>0$, then the unstable manifold $W^{u}(O)$ is on the outside boundary of $P_{\rho}$ (inward twist case), and if $g_{X}^{1}(0,0 ; 0) g_{Y Y}^{1}(0,0 ; 0)<0$, then the unstable manifold is on the inside boundary of $P_{\rho}$ (outward twist case). In what follows, $g_{Y Y}^{1}(0,0 ; 0)$ is assumed to be positive. (See Fig 2.2.) 
2.2. Bifurcation of $n$-homoclinic orbits. Next, a part of the results of Homburg et al concerning existence of $n$-homoclinic orbits and a brief sketch of the correspondence between symbolic sequences and the orbits are summarized.

The followings is the relevant part of the theorem in Homburg Kokubu and Krupa [9].

Proposition 2.1. Consider a two parameter family of equations $u^{\prime}=\mathfrak{X}_{\nu}(u)$ having an inclination-flip bifurcation point at $\nu=0$ and satisfying the assumptions in the previous section.

For the case of inward twist, there exist functions $\nu_{1}^{-}\left(\nu_{2}\right)<0<\nu_{1}^{+}\left(\nu_{2}\right)$, a neighborhood $\mathcal{U}$ of $\bar{\Gamma}$ and $\varepsilon>0$ such that for each $-\varepsilon<\nu_{2}<0$ the following statements hold.

(i) When $0<\nu_{1}<\nu_{1}^{+}\left(\nu_{2}\right)$ the non-wandering set in $\mathcal{U}$ is the union of the singularity at $O$ and a suspended horseshoe, namely the Poincaré map along $\Gamma$ possesses a horseshoe.

(ii) As $\nu_{1}$ decreases from 0 to $\nu_{1}^{-}\left(\nu_{2}\right)$, all the orbits of the suspended horseshoe disappear in a bifurcation connecting to the origin $O$. Especially, periodic orbits disappear in infinite period bifurcations generating homoclinic orbits.

(iii) There exists a symbolic representation of periodic orbits and homoclinic orbits which bifurcate from the periodic orbits.

For the case of outward twist, there exist functions $0<\nu_{1}^{-}\left(\nu_{2}\right)<\nu_{1}^{+}\left(\nu_{2}\right)$, a neighborhood $\mathcal{U}$ of $\bar{\Gamma}$ and $\varepsilon>0$ such that for each $-\varepsilon<\nu_{2}<0$ the following statements hold.

(i) When $\nu_{1}^{-}\left(\nu_{2}\right)<\nu_{1}<\nu_{1}^{+}\left(\nu_{2}\right)$ the non-wandering set in $\mathcal{U}$ is the union of the singularity at $O$ and a suspended horseshoe.

(ii) As $\nu_{1}$ decreases from $\nu_{1}^{-}\left(\nu_{2}\right)$ to 0 , all the orbits of the suspended horseshoe disappear in a bifurcation connecting to the origin $O$. Especially, periodic orbits disappear in infinite period bifurcations generating homoclinic orbits.

(iii) There exists a symbolic representation of periodic orbits and homoclinic orbits which bifurcate from the periodic orbits.

Above result is proven by constructing an invariant foliation and reducing analysis of the return map $f_{\nu}$ to analysis of a multi-valued one dimensional map. This reduction and the correspondence between periodic or homoclinic orbits and symbolic sequences are explained in the sequel. For this purpose, the local map $F$ is decomposed into two parts:

$$
\begin{aligned}
& S_{\delta}: \Sigma_{1} \rightarrow \Sigma_{\delta}:(1, y, z) \mapsto\left(\delta, \delta^{\frac{\lambda^{u u}}{\lambda^{s}}} y, \delta^{\frac{\lambda^{u}}{\lambda^{s}}} z\right) \\
& F_{\delta}: \Sigma_{\delta} \rightarrow \Sigma_{0}:(\delta, y, z) \mapsto\left(\delta z^{-\frac{\lambda^{s}}{\lambda^{u}}}, y z^{-\frac{\lambda^{u u}}{\lambda^{u}}}, 1\right)
\end{aligned}
$$

where $\Sigma_{\delta}:=\{x=\delta,|y|,|z|<1\}$, and the return map from $C_{\rho, \delta}:=F_{\delta}^{-1}\left(R_{\rho}\right)$ to itself is put under consideration. This map is also denoted as $f_{\nu}: C_{\rho, \delta} \rightarrow C_{\rho, \delta}$.

First, existence of invariant foliation is stated below.

Proposition 2.2. Let $R$ and $D$ be constants satisfying

$$
2<R<-\frac{\lambda^{s}}{\lambda^{u}}, \quad \max \left\{\frac{\lambda^{s}}{\lambda^{u}-\lambda^{u u}},-\frac{2 \lambda^{s}}{\lambda^{u u}}\right\}<D<R
$$

and $\alpha:=\frac{\lambda^{u}-\lambda^{u u}}{\lambda^{s}}$, and $\mathcal{P}:=\left\{-\varepsilon<\nu_{2}<0,\left|\nu_{1}\right|<\left|\nu_{2}\right|^{2+\kappa}\right\}$ for some $0<\kappa<R-2$ and $\varepsilon>0$, where $\varepsilon$ depends on $\kappa$. (The dependence is specified in [9].) 


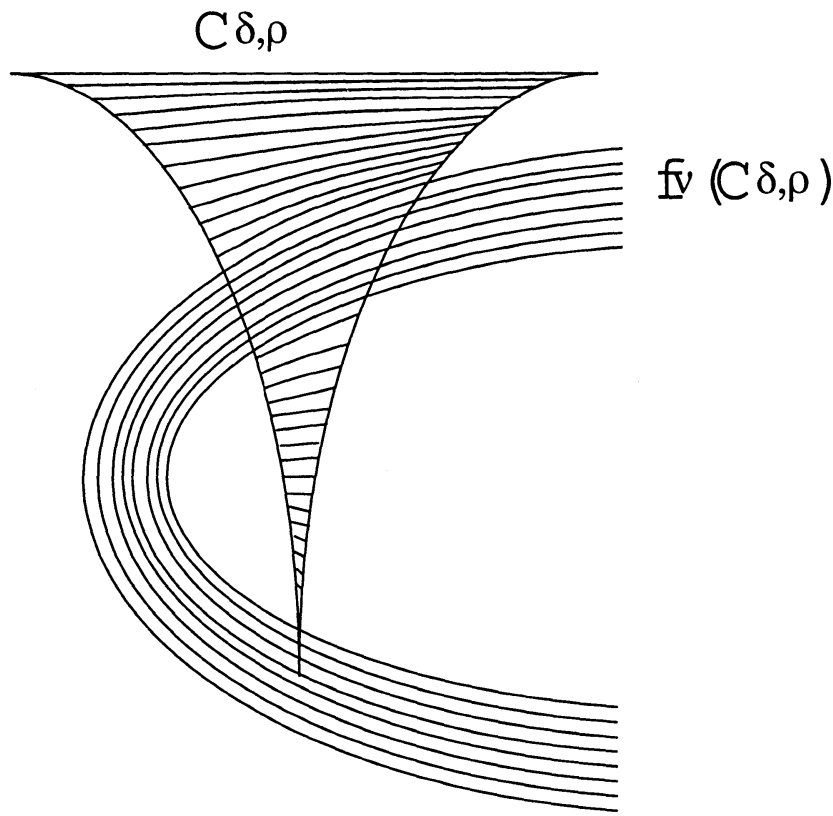

FIG. 2.3.

Then, for $\rho=\left|\nu_{2}\right|^{R}, \delta=\left|\nu_{2}\right|^{D}$ and $\nu \in \mathcal{P}$ there exists a foliation $\mathcal{F}^{u}$ on the cusp $C_{\rho, \delta}$ satisfying the following properties. (See Fig. 2.3.)

(A) $\mathcal{F}^{u}$ is invariant for $f$ in the following sense. If $l$ is a leaf of $\mathcal{F}^{u}$ then the connected components of $f(l) \cap C_{\rho, \delta}$ are leaves of $\mathcal{F}^{u}$.

(B) $\mathcal{F}^{u}$ depends $C^{1+\alpha}$-smoothly on the base points and on the parameter $\nu_{1}$. The dependence on $\nu_{2}$ is continuous. The leaves of $\mathcal{F}^{u}$ are at least $C^{2}$ smooth.

(C) $f$ contracts distances between the leaves of $\mathcal{F}^{u}$ and expands distances along the leaves of $\mathcal{F}^{u}$. More precisely there exists $\eta>1$ such that

1. if $f\left(l_{1}\right)$ and $f\left(l_{2}\right)$ are in the same connected component of $f\left(C_{\rho, \delta}\right) \cap C_{\rho, \delta}$ then $\operatorname{dis}\left(f\left(l_{1}\right), f\left(l_{2}\right)\right)<\eta^{-1} \cdot \operatorname{dis}\left(l_{1}, l_{2}\right)$,

2. if $x, y \in l$ and $f(x), f(y) \in C_{\rho, \delta}$, then $\operatorname{dis}(f(x), f(y))>\eta \cdot \operatorname{dis}(x, y)$.

With this foliation, an one-dimensional multi-valued map $\pi_{\nu}$ is defined as follows. Let $I:=\left(0, t_{\nu}\right]$ be the intersecting part of the line $\{y=0\} \subset \Sigma_{\delta}$ with the cusp $C_{\rho, \delta}$, where $t_{\nu}:=\left|\nu_{2}\right|^{(D-R) \frac{\lambda^{u}}{\lambda^{s}}}$, and $\tau$ be the projection of the leaves of $\mathcal{F}^{u}$ onto $I$. Then the multi-valued map $\pi_{\nu}$ is defined as

$$
\pi_{\nu}: I \rightarrow I: z \mapsto \tau \circ f_{\nu} \circ \tau^{-1}(z)
$$

This map is the union of two $C^{1+\alpha}$ maps $\xi_{\nu}(z)$ and $\eta_{\nu}(z)$ satisfying $\xi_{\nu}(z)>\eta_{\nu}(z)$ where both are defined - in fact, the domain of $\eta_{\nu}$ is a subinterval $K_{\nu}$ of $I$. Although $\pi_{\nu}$ is defined on $\left(0, t_{\nu}\right]$, it is extended to $z=0$ by requiring that $\pi_{\nu}(0)=\lim _{z \rightarrow 0} \pi_{\nu}(z)$. Note that each trajectory of $\pi_{\nu}$ remaining in $I$ corresponds to a unique trajectory of $\left.f_{\nu}\right|_{C_{\rho, \delta}}$ and each trajectory of $f_{\nu}$ which remains in $C_{\rho, \delta}$ for all positive time corresponds to a trajectory of $\pi_{\nu}$.

This $\pi_{\nu}$ is characterized by the next lemma. 


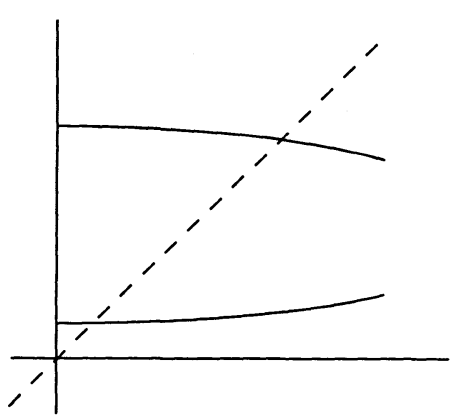

$$
v_{1}>0
$$

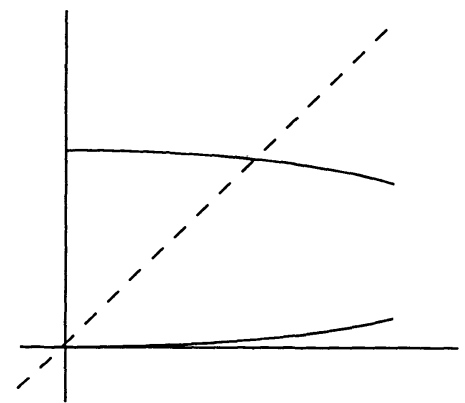

$$
v_{1}>0
$$

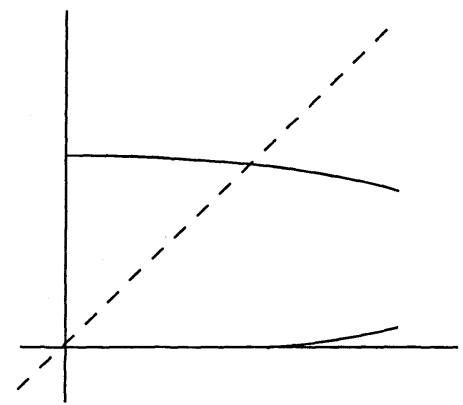

\section{$v_{1}<0$}

FIG. 2.4

LEMMA 2.1. In the case of inward twist,

1. if $\nu_{1}>0$ then $K_{\nu}=\left[0, t_{\nu}\right]$ and $\eta_{\nu}(0)>0$,

2. if $\nu_{1}=0$ then $K_{\nu}=\left[0, t_{\nu}\right]$ and $\eta_{\nu}(0)=0$,

3. if $\nu_{1}<0$ then $K_{\nu}=\left[a_{\nu}, t_{\nu}\right]$ with $a_{\nu}=\eta^{-1}(0)>0$, and $K_{\nu}$ shrinks as $\nu_{1}$ decreases. Moreover, there exists $\nu_{1}^{-}\left(\nu_{2}\right)<0$ so that if $\nu_{1}<\nu_{1}^{-}\left(\nu_{2}\right)$ then $K_{\nu}=\emptyset$.

$\xi_{\nu}^{\prime}(z)<0$ and $\eta_{\nu}^{\prime}(z)>0$ holds for $\nu_{1}>\nu_{1}^{-}\left(\nu_{2}\right)$. (See Fig. 2.4.)

In the case of outward twist, there exists $\nu_{1}^{-}\left(\nu_{2}\right)>0$ so that

1. if $\nu_{1}>\nu_{1}^{-}\left(\nu_{2}\right)$ then $K_{\nu}=\left[0, t_{\nu}\right]$ and $\eta_{\nu}\left(t_{\nu}\right)>0$,

2. if $\nu_{1}=\nu_{1}^{-}\left(\nu_{2}\right)$ then $K_{\nu}=\left[0, t_{\nu}\right]$ and $\eta_{\nu}\left(t_{\nu}\right)=0$,

3. if $\nu_{1}<\nu_{1}^{-}\left(\nu_{2}\right)$ then $K_{\nu}=\left[0, b_{\nu}\right]$ with $b_{\nu}:=\eta_{\nu}^{-1}(0)$ and $K_{\nu}$ shrinks as $\nu_{1}$ decreases. Moreover, if $\nu_{1}<0$ then $K_{\nu}=\emptyset$.

$\xi_{\nu}^{\prime}(z)>0$ and $\eta_{\nu}^{\prime}(z)<0$ holds for $\nu_{1}>0$. (See Fig.6.) 

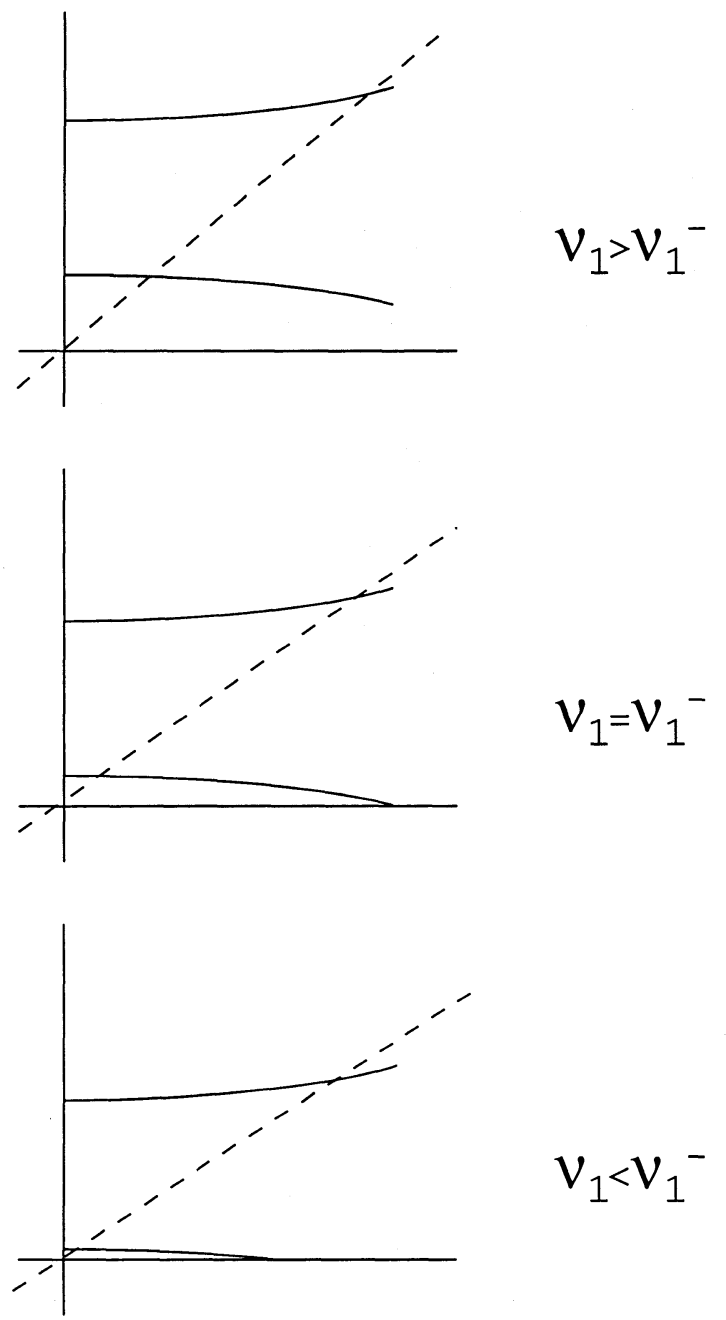

FIG. 2.5.

Following [9], the inward twist case is focused on for a moment and later outward twist case is mentioned.

As $\pi_{\nu}^{-1}$ is single valued map well defined on the union of two closed subinterval of $I$, symbolic dynamics for $\pi_{\nu}^{-1}$ can be defined in the similar manner for quadratic map. For each $x \in I$ with the property that $\pi_{\nu}^{-j}(x) \in I$ for all $j \in \mathbb{N}$, the sequence $S(x)=\left(S_{0}(x), S_{1}(x), \cdots\right)$ of the letters -1 and +1 are defined as

$$
S_{j}(x)= \begin{cases}-1 & \text { if } \pi_{\nu}^{-j}(x) \in \xi_{\nu}(I) \\ +1 & \text { if } \pi_{\nu}^{-j}(x) \in \eta_{\nu}(I)\end{cases}
$$

If $\sigma=\left(\sigma_{0}, \sigma_{1}, \cdots\right)$ and $\tau=\left(\tau_{0}, \tau_{1}, \cdots\right)$ are such symbols, then $\sigma \prec \tau$ provided that $\sigma_{i}=\tau_{i}$ for $0 \leq i \leq j-1$ and

$$
\begin{cases}\sigma_{0} \cdot \sigma_{1} \cdots \sigma_{j-1}=+1, & \sigma_{j}=+1 \text { and } \tau_{j}=-1 \\ \sigma_{0} \cdot \sigma_{1} \cdots \sigma_{j-1}=-1, & \sigma_{j}=-1 \text { and } \tau_{j}=+1\end{cases}
$$


Note that each periodic orbit of $\pi_{\nu}^{-1}$ of period $n$ has $n$ different symbolic sequences corresponding to the $n$ different initial points. The minimal sequence in them is referred to as the sequence of the periodic orbit.

The $n$-homoclinic orbits, which are of interest in this paper, are generated in the process of disappearance of these periodic orbits. More precisely, when $\nu_{2}$ is fixed at a small value and $\nu_{1}$ is decreased from 0 , the leftmost point in each periodic orbit approaches 0 , and the orbit disappears when it passes 0 . A periodic orbit of $\pi_{\nu}$ which involves 0 in its orbit corresponds to a homoclinic orbit of the vector field, and disappearance of a periodic orbit corresponds to disappearance of a periodic orbit of vector field through an infinite period bifurcation generating a homoclinic orbit.

The case of outward twist is reduced to inward twist case. This is carried out through conjugacy map $h:\left[0, b_{\nu}\right] \rightarrow[0,1]$ which is the composition of reflection in the midpoint and rescaling, i.e. $\tilde{\pi}_{\nu}:=h \circ \pi_{\nu} \circ h^{-1}$ satisfies conditions required for inward twist case. Consequently, the same as above holds. In this case, however, the role of -1 and +1 is interchanged because of the reflection, moreover, a symbolic sequence $\sigma=\left(\sigma_{1}, \cdots, \sigma_{n-1}, \sigma_{0}\right)^{\infty}$ for $\tilde{\pi}_{\nu}$ corresponds to the sequence $\sigma=\left(\sigma_{0}, \sigma_{1}, \cdots, \sigma_{n-1}\right)^{\infty}$ for $\pi_{\nu}$.

3. The theorem. The main theorem shall be stated in this section. Namely, distribution of the real eigenvalues of the linear stability problem associated with each $n$-pulse is treated.

3.1. The eigenvalue problem. First, the setting of the problem is introduced. Consider the equation of traveling wave on $\mathbb{R}^{3}$ :

$$
u^{\prime}=\mathfrak{X}(u ; \nu) \quad \nu \in \mathbb{R}^{2}
$$

and assume that the system satisfies the conditions in the previous section. Assume also that the local coordinates $(x, y, z)$ near the origin is chosen so that (2.3) holds and the parameter $\nu$ is taken as in (2.5) and belongs to $\mathcal{P}$. In the sequel, a condition stronger than (EV) is assumed because of technical reasons:

EV': $\lambda^{u u}>3 \lambda^{u}$ and $-\lambda^{s}>2 \lambda^{u}$.

Under this situation, the equilibrium $u \equiv O$ corresponds to a steady state solution and the homoclinic orbit $\Gamma$ to a single pulse solution, moreover existence of $n$-homoclinic orbits is equivalent to existence of $n$-pulse solutions.

Let $u=h(t)$ be a homoclinic solution to $O$-either the original one-homoclinic solution or one of the bifurcating $n$-homoclinic solutions-then linearized eigenvalue problem along $h(t)$ is written in the following form.

$$
v^{\prime}=[D \mathfrak{X}(h(t) ; \nu)+\Lambda B] v
$$

where $B$ is some constant matrix. A non-trivial solution of this equation is an eigenfunction if and only if it is bounded, and the $\Lambda$ at which such a solution exists is an eigenvalue.

REMARK 3.1. For general eigenvalue problem, $A$ is in some domain of complex plane. However, it is assumed to be real throughout this paper as the geometric relation of (3.1) and (3.2) is the matter of interest.

3.2. Geometric interpretation of the eigenvalue problem. Before the result is stated, geometric interpretation of the eigenvalue problem needs to be explained. 
In order to do this, an additional coordinate change is applied to (3.2). Due to the assumption (2.3), the eigenvalue problem is written in the following form when $h(t)$ is in $\mathcal{N}$.

$$
\left(\begin{array}{l}
v_{1}{ }^{\prime} \\
v_{2}^{\prime} \\
v_{3}^{\prime}
\end{array}\right)=\left[\left(\begin{array}{ccc}
\lambda_{\nu}^{s} & 0 & 0 \\
0 & \lambda_{\nu}^{u u} & 0 \\
0 & 0 & \lambda_{\nu}^{u}
\end{array}\right)+\Lambda B\right]\left(\begin{array}{l}
v_{1} \\
v_{2} \\
v_{3}
\end{array}\right)
$$

Through a smooth coordinate change, this is again diagonalized for small $|\Lambda|$ :

$$
\left(\begin{array}{l}
v_{1}^{\prime} \\
v_{2}^{\prime} \\
v_{3}^{\prime}
\end{array}\right)=\left(\begin{array}{ccc}
\lambda_{\nu}^{s}(\Lambda) & 0 & 0 \\
0 & \lambda_{\nu}^{u u}(\Lambda) & 0 \\
0 & 0 & \lambda_{\nu}^{u}(\Lambda)
\end{array}\right)\left(\begin{array}{l}
v_{1} \\
v_{2} \\
v_{3}
\end{array}\right)
$$

where $\lambda_{\nu}^{*}(0)=\lambda_{\nu}^{*}$ for $*=s, u u$ or $u$.

Geometric meaning of the eigenvalue problem is easily seen in these coordinates. That is, suppose that $v(t)$ is a non-trivial solution and it is expressed as $v(t)=$ $\left(v_{1}(t), v_{2}(t), v_{3}(t)\right)$ when $t \approx \pm \infty$ and thus $h(t)$ is in $\mathcal{N}$, then $v(t)$ is bounded if and only if $v_{2}(t)=v_{3}(t)=0$ for $t \approx+\infty$ and $v_{1}(t)=0$ for $t \approx-\infty$. In the sequel, $h(t)$ is assumed to be the solution which hits $\Sigma_{1}$ (for the last time if $h(t)$ is $n$-homoclinic solution) at $t=0$ to avoid ambiguity, and the solution $v(t ; \Lambda)$ of $(3.2)$ with initial condition $\left(v_{1}(0 ; \Lambda), v_{2}(0 ; \Lambda), v_{3}(0 ; \Lambda)\right)=(-1,0,0)$ in the coordinates of $(3.3)$ is traced. Then the fact above is expressed as follows.

LEMMA 3.1. $\Lambda$ is an eigenvalue if and only if $v_{1}(t ; \Lambda)=0$ for $t \approx-\infty$.

The analysis requires a non-degeneracy condition on the eigenvalue problem (3.2). Let $h_{0}(t)$ be the original homoclinic solution for $\nu=0$ and let $t_{0}$ be the time when $h_{0}(t)$ hits the section $\Sigma_{0}$, then the solution $v_{0}(t, \Lambda)$ with the initial condition $\left(v_{01}(0 ; \Lambda), v_{02}(0 ; \Lambda), v_{03}(0 ; \Lambda)\right)=(-1,0,0)$ is assumed to satisfy the following nondegeneracy condition.

ND: $D:=\frac{\partial v_{01}\left(t_{0} ; 0\right)}{\partial \Lambda} \neq 0$

REMARK 3.2. $v_{01}\left(t_{0} ; 0\right)=0$ as $\Lambda=0$ is an eigenvalue corresponding to the spatial translation of the pulse.

3.3. Statement of the theorem. Now the theorem is ready to be stated.

Let $\sigma=\left(\sigma_{0}, \cdots, \sigma_{n-1}\right)^{\infty}$ be a minimal periodic symbolic sequence, then as is presented in the previous section there is a $n$-homoclinic orbit $h_{\sigma}(t)$ which is generated from the periodic orbit in a infinite period bifurcation. A number $N(\sigma)$ for the homoclinic orbit $h_{\sigma}(t)$ is defined as follows.

DEFINITION 3.1 .

$$
N(\sigma):=\mid 1+\sum_{k=1}^{n-2} \sigma_{1} \cdot \sigma_{2} \cdots \sigma_{k}+\left\{\begin{array}{ccc}
-1 & \text { if } & \sigma_{1} \cdots \sigma_{n-1}=-1 \\
0 & \text { if } & \sigma_{1} \cdots \sigma_{n-1}=+1
\end{array} \mid\right.
$$

Concerning the eigenvalue problem associated to $h_{\sigma}(t)$ the following holds.

Theorem. Assume that $D<0(D>0)$. Then, the eigenvalue problem (3.2) for $h=h_{\sigma}$ has at least $N(\sigma)$ negative (positive) eigenvalues if $\left|\nu_{2}\right|$ is sufficiently small.

COROLlary. In the case of outward twist, the multiple pulse solutions corresponding to symbolic sequences $(-1,+1 \cdots+1$ ) (all symbols are +1 except the first one) are stable for sufficiently small $\left|\nu_{2}\right|$ if the original 1-pulse is stable. 
4. The index. The proof of the theorem depends on a topological method, namely defining an index which counts the number of eigenvalues. The purpose of this section is to define this index.

4.1. Existence of confining cone. The index is constructed through restricting the eigenvalue problem (3.3) in the three dimensional space to a two dimensional cone. Finding this cone is the first task.

Let $h_{\sigma}(t)$ be one of the $n$-homoclinic orbit and $v(t ; \Lambda)$ be the solution of (3.3) with initial condition $\left(v_{1}(0 ; \Lambda), v_{2}(0 ; \Lambda), v_{3}(0 ; \Lambda)\right)=(-1,0,0)$, and denote the time at which $h_{\sigma}(t)$ hits $R_{\rho}$ for the $k$-th times as $T_{n-k+1}$. Because the eigenvalues converges to 0 in the limit of $\nu_{2} \rightarrow 0$, the parameter $\Lambda$ is rescaled as $\Lambda=\left|\nu_{2}\right|^{\chi} \tilde{\Lambda}$ for some fixed $\chi$ satisfying $1<\chi<\frac{3}{2}$. Then the following is proven.

Proposition 4.1. Assume that the constant $R$ appeared in proposition2.2 satisfies $\frac{2 \lambda^{s}}{\lambda^{u}-\lambda^{u u}}<R$.

Then, there exist $c>0$ and $\tilde{\Lambda}_{0}>0$ so that $\left(v_{1}\left(T_{k} ; \Lambda\right), v_{2}\left(T_{k} ; \Lambda\right), v_{3}\left(T_{k} ; \Lambda\right)\right)$ is inside the cone $\mathcal{C}:=\left\{V\left|\sqrt{\left|V_{1}\right|^{2}+\left|V_{3}\right|^{2}}>c\right| \nu_{2}|| V_{2} \mid\right\}$ if $|\tilde{\Lambda}|<\tilde{\Lambda}_{0}$ for sufficiently small $\left|\nu_{2}\right|$.

REMARK 4.1. Condition EV' is necessary to choose $R$ satisfying the condition above.

If $u(t)$ is a solution of (3.1) staying in the cube $\{|x|,|y|,|z| \leq 1\}$ for $t_{1} \leq t \leq t_{2}$ which starts in $C_{\rho}$ at $t=t_{1}$ and reaches $R_{\rho}$ at $t=t_{2}$, then a straight forward calculation taking into account that $\rho=\left|\nu_{2}\right|^{R}$ under the condition $\frac{2 \lambda^{s}}{\lambda^{u}-\lambda^{u u}}<R$ shows the following.

LEMMA 4.1. For arbitrary fixed $c, c^{\prime}>0$ and $\chi^{\prime}>1$ with $1<\chi^{\prime}<\frac{\lambda^{u}-\lambda^{u u}}{\lambda^{s}} R-1$, there exists $\tilde{\Lambda}_{1}>0$ so that the following holds. Any solution to (3.3) with the initial condition inside the cone $\sqrt{\left|V_{1}\right|^{2}+\left|V_{3}\right|^{2}} \geq c\left|\nu_{2}\right|\left|V_{2}\right|$ at $t=t_{2}$ is inside the cone $\left|\nu_{2}\right|^{\chi^{\prime}} \sqrt{\left|v_{1}\right|^{2}+\left|v_{3}\right|^{2}}>c^{\prime}\left|v_{2}\right|$ at $t=t_{1}$ if $|\tilde{\Lambda}|<\tilde{\Lambda}_{1}$ for sufficiently small $\left|\nu_{2}\right|$.

On the other hand, if $u(t)$ is a solution of (3.1) which starts in $R_{\rho}$ at $t=t_{1}$ and reaches $C_{\rho} \cap P_{\rho}$ at $t=t_{2}$ and stay outside of the cube $\{|x|,|y|,|z| \leq 1\}$ for $t_{1}<t<t_{2}$, then the following holds.

LEMMA 4.2. For any $\chi>1$, there exist $c, c^{\prime}>0$ and $\tilde{\Lambda}_{2}>0$ so that any solution of (3.3) with the initial condition inside the cone $\left|\nu_{2}\right|^{\chi} \sqrt{\left|v_{1}\right|^{2}+\left|v_{3}\right|^{2}}>c^{\prime}\left|v_{2}\right|$ at $t=t_{2}$ is inside the cone $\sqrt{\left|V_{1}\right|^{2}+\left|V_{3}\right|^{2}}>c\left|\nu_{2}\right|\left|V_{2}\right|$ at $t=t_{1}$ if $|\tilde{\Lambda}|<\left|\tilde{\Lambda}_{2}\right|$ for sufficiently small $\left|\nu_{2}\right|$.

Proof of this lemma is divided into three steps.

First, the Poincaré map $G_{\nu}$ has the following property.

Claim 4.1. For $(y, z) \in C_{\rho} \cap P_{\rho}$, let $\bar{v}=\left(\bar{v}_{2}, \bar{v}_{3}\right) \in T_{(y, z)} \Sigma_{1}$ and $\bar{V}=\left(\bar{V}_{1}, \bar{V}_{2}\right)=$ $D G_{\nu}^{-1} \bar{v} \in T_{G_{\nu}^{-1}(y, z)} \Sigma_{0}$. Then for any $c_{1}>0$ there exists $c_{2}>0$ independent of $(y, z)$ so that $\bar{V}$ satisfies $\left|V_{1}\right|>c_{2}\left|\nu_{2}\right|\left|V_{2}\right|$ if $\bar{v}$ satisfies $c_{1}\left|\nu_{2}\right| \chi\left|\bar{v}_{3}\right|>\left|\bar{v}_{2}\right|$.

Proof. A foliation on the parabola-like region $P_{\rho}$ is defined to analyze the composition of the parabola and the cone $C_{\rho}$. Consider a segment $\{\xi\} \times[-1,+1] \subset R_{\rho}$ for $0 \leq \xi \leq \rho$. This segment is mapped to a curve on $\Sigma_{1}$ written by

$$
\left\{\begin{array}{l}
y=g^{1}(\xi, Y ; \nu) \\
z=g^{2}(\xi, Y ; \nu)
\end{array} \quad-1 \leq Y \leq 1\right.
$$


(2.4) means that the second equation can be solved for $Y$ with

$$
g^{2}(\xi, Y(z ; \xi, \nu) ; \nu)=z \text { and } Y(0 ; 0,0)=0
$$

Then, the graph $y=\varphi(z ; \xi, \nu)$ of the function $\varphi$ defined by

$$
\varphi(z ; \xi, \nu)=g^{1}(\xi, Y(z ; \xi, \nu) ; \nu)
$$

gives a leaf of this foliation. Moreover, this $\varphi$ is expanded as follows.

$$
\varphi(z ; \xi, \nu)=\nu_{1}+\nu_{2} z+a z^{2}+b \xi+O\left(|\xi|^{2},|z|^{3},|\nu|^{2}\right)
$$

Here, when $\nu=0, a$ is equal to $\frac{g_{Y Y}^{1}(0,0 ; 0)}{\left\{g_{Y}^{2}(0,0 ; 0)\right\}^{2}}$ and thus positive, whereas $b$ is equal to $g_{X}^{1}(0,0 ; 0)$ and hence $b>0$ in inward twist case and $b<0$ in outward twist case.

The claim follows from the fact that $G_{\nu}$ is diffeomorphism and that there exists constant $c_{3}>0$ so that

$$
\left|\frac{\partial \varphi}{\partial z}(z ; \xi, \nu)\right|>c_{3}\left|\nu_{2}\right|
$$

holds if $(\varphi(z ; \xi, \nu), z) \in C_{\rho} \cap P_{\rho}$ (See proposition 2 in [9]).

ClaIM 4.2. There exist $c_{4}>0$ and $c_{5}>0$ so that any solution to (3.3) for $\Lambda=0$ with the initial condition inside the cone $\left|\nu_{2}\right|^{\chi} \sqrt{\left|v_{1}\right|^{2}+\left|v_{3}\right|^{2}}>c_{4}\left|v_{2}\right|$ at $t=t_{2}$ is inside the cone $\sqrt{\left|V_{1}\right|^{2}+\left|V_{3}\right|^{2}}>c_{5}\left|\nu_{2}\right|\left|V_{2}\right|$ at $t=t_{1}$.

Proof. The tangent vector $\mathfrak{X}(u(t) ; \nu)$ to the solution $u(t)$ satisfies (3.3) for $\Lambda=0$ and it is expressed as

$$
\begin{aligned}
\mathfrak{X}\left(u\left(t_{1}\right) ; \nu\right) & =\left(\lambda_{\nu}^{s} X, \lambda_{\nu}^{u u} Y, \lambda_{\nu}^{u}\right) \\
\text { and } & \\
\mathfrak{X}\left(u\left(t_{2}\right) ; \nu\right) & =\left(\lambda_{\nu}^{s}, \lambda_{\nu}^{u u} y, \lambda_{\nu}^{u} z\right)
\end{aligned}
$$

where $u\left(t_{1}\right)=(X, Y, 1)$ and $u\left(t_{2}\right)=(1, y, z) .(1, y, z) \in C_{\rho} \cap P_{\rho}$ implies $z=O\left(\left|\nu_{2}\right|\right)$ and $y=O\left(\left|\nu_{2}\right|^{\frac{\lambda^{u u}}{\lambda^{u}}}\right)$ (see proposition 2 in [9]). Moreover $X=O\left(\left|\nu_{2}\right|^{R}\right)$ and $Y=O\left(\left|\nu_{2}\right|\right)$ are easily seen. Therefore, there exists $c_{6}>0$ so that $\sqrt{\left(\left|V_{1}\right|^{2}+\left|V_{3}\right|^{2}\right)}>c_{5}\left|\nu_{2}\right|\left|V_{2}\right|$ holds if $\left|v_{1}\right|>c_{6} \sqrt{\left|v_{2}\right|^{2}+\left|v_{3}\right|^{2}}$ is satisfied.

On the other hand, claim 4.1 implies that there exists $c_{7}>0$ so that any solution with the initial condition inside the cone $\left|\nu_{2}\right| \chi\left|v_{3}\right|>c_{7}\left|v_{2}\right|$ at $t=t_{2}$ is inside the cone $\sqrt{\left|V_{1}\right|^{2}+\left|V_{3}\right|^{2}}>c_{5}\left|\nu_{2}\right|\left|V_{2}\right|$ at $t=t_{1}$. Then the claim is easily obtained.

Proof. (Proof of lemma 4.2) The lemma immediately follows from the claim above and the fact that $\Lambda=\left|\nu_{2}\right|^{\chi} \tilde{\Lambda}$ with $\chi>1$.

Proof. (Proof of proposition 4.1) The lemma 4.2 means that the proposition holds for $t=T_{1}$. Successive application of lemma 4.1 and lemma 4.2 shows the proposition for $t=T_{k}, k \geq 2$.

The basis of the index is the fact that the set $\mathcal{C} \subset \mathbb{R}^{3}$ has one-dimensional homology group isomorphic to $\mathbb{Z}$. In the sequel, the problem is dealt with in the projective space $\mathbb{R P}^{2}$ rather than $\mathbb{R}^{3}$ to suppress the ambiguity of multiplication of constant.

Consider the coupled system of (3.1) and (3.2)

$$
\left\{\begin{array}{l}
u^{\prime}=\mathfrak{X}(u ; \nu) \\
v^{\prime}=[D \mathfrak{X}(h(t) ; \nu)+\Lambda B] v
\end{array}\right.
$$


on $\bar{\Gamma}_{\sigma} \times \mathbb{R}^{3}$, where $\Gamma_{\sigma}:=\left\{h_{\sigma}(t) \mid-\infty<t<+\infty\right\}$ and $\bar{\Gamma}_{\sigma}=\{O\} \cup \Gamma_{\sigma}$. Here, through suitable coordinate change, $v$-component of the system is assumed to be of the form of (3.3) as far as $u(t)$ is in $\mathcal{N}$. As this system is linear in $v$-component, the flow defined by it induces a flow on $\bar{\Gamma}_{\sigma} \times \mathbb{R P}^{2}$ in the following way.

Let $\Phi^{t}: \bar{\Gamma}_{\sigma} \times \mathbb{R}^{3} \rightarrow \bar{\Gamma}_{\sigma} \times \mathbb{R}^{3}$ be the flow determined by (4.2) and $\Pi: \mathbb{R}^{3} \rightarrow \mathbb{R P}^{2}$ be the projection. Then, the flow $\hat{\Phi}^{t}$ on $\bar{\Gamma}_{\sigma} \times \mathbb{R} \mathbb{P}^{2}$ is defined by

$$
\hat{\Phi}^{t}(u, \hat{v}):=(\mathrm{id} \times \Pi)\left(\Phi^{t}(u, v)\right)
$$

where $\hat{v}:=\Pi(v)$. This definition does not depend on the choice of $v \in \Pi^{-1}(\hat{v})$ as the flow $\Phi^{t}$ is linear in $v$.

In this framework, lemma 3.1 is interpreted as follows.

LEMMA 4.3. $\Lambda$ is an eigenvalue if and only if the orbit $\left(h_{\sigma}(t), \hat{v}(t ; \Lambda)\right)$ of the flow $\hat{\Phi}^{t}$ which converges to $(O,[-1: 0: 0])$ in the limit of $t \rightarrow+\infty$ converges to $(O,[0: 1: 0])$ or $(O,[0: 0: 1])$ in the limit of $t \rightarrow-\infty$.

On the other hand, if $\Lambda$ is not an eigenvalue, $\left(h_{\sigma}(t), \hat{v}(t ; \Lambda)\right)$ converges to $(O,[1$ : $0: 0])$ as $t \rightarrow-\infty$.

Next task is interpretation of proposition 4.1 in this framework. Let $\widehat{\mathcal{C} \Gamma_{0}}, \widehat{\mathcal{C} \Gamma_{k}}$ $(k=1, \cdots n-1)$ and $\widehat{\mathcal{C} \Gamma_{n}}$ be defined as

$$
\begin{aligned}
& \widehat{\mathcal{C}}_{0}:=\overline{\left\{\hat{\Phi}^{t}(\gamma, \hat{v}) \mid \gamma=h_{\sigma}(0), \hat{v}=\left[v_{1}: 0: v_{3}\right], T_{1} \leq t<+\infty\right\}} \\
& \widehat{\mathcal{C}}_{k}:=\left\{\hat{\Phi}^{t}(\gamma, \hat{v}) \mid \gamma=h_{\sigma}\left(T_{k}\right), \hat{v} \in \hat{\mathcal{C}}, T_{k+1}-T_{k} \leq t \leq 0\right\} \\
& \widehat{\mathcal{C}}_{n}:=\overline{\left\{\hat{\Phi}^{t}(\gamma, \hat{v}) \mid \gamma=h_{\sigma}\left(T_{n}\right), \hat{v} \in \hat{\mathcal{C}},-\infty<t \leq 0\right\}}
\end{aligned}
$$

where $\hat{\mathcal{C}}:=\Pi(\mathcal{C})$ with $\mathcal{C}=\left\{v\left|\sqrt{\left|V_{1}\right|^{2}+\left|V_{3}\right|^{2}}>c\right| \nu_{2}|| V_{2} \mid\right\}$ which appeared in proposition 4.1 and $\overline{\{\}}$ stands for the closure of the set. Then by lemma 4.1 and lemma 4.2 , $\widehat{\mathcal{C} \Gamma}:=\bigcup_{k=0}^{n} \widehat{\mathcal{C} \Gamma}_{k}$ is homotopic to $\bar{\Gamma}_{\sigma} \times \mathbb{S}^{1}$ for each $\Lambda$. Moreover, the following is easily seen.

Proposition 4.2. There are an open set $S_{0} \subset \mathbb{R P}^{2}$ which is homotopic to $\mathbb{S}^{1}$ and a subset $\mathcal{S}$ of $\bar{\Gamma}_{\sigma} \times \mathbb{R P}^{2}$ which is homeomorphic to $\bar{\Gamma}_{\sigma} \times S_{0}$ with the following property.

1. $\mathcal{S} \supset \widehat{\mathcal{C}} \Gamma$ if $\Lambda<\left|\nu_{2}\right|{ }^{\chi} \tilde{\Lambda}_{0}$.

2. There is a homeomorphism $\Xi: \mathcal{S} \rightarrow \bar{\Gamma}_{\sigma} \times S_{0}$ satisfying $\Xi\left(\mathcal{S} \cap\left(\{\gamma\} \times \mathbb{R} \mathbb{P}^{2}\right)\right)=$ $\{\gamma\} \times S_{0}$ for each $\gamma$.

4.2. Definition of the index. Finally the index is defined in this subsection.

Take $\Lambda_{1}$ and $\Lambda_{2}\left(\Lambda_{1}<\Lambda_{2}\right)$ satisfying $\left|\Lambda_{i}\right|<\left|\nu_{2}\right| \chi \tilde{\Lambda}_{0}$ and neither of which is an eigenvalue. Then a map $\mathfrak{g}: \partial\left(\left[\lambda_{1}, \lambda_{2}\right] \times[-1,+1]\right) \rightarrow S_{0}$ defined below is continuous.

$\mathfrak{g}(\Lambda, \tau)= \begin{cases}\operatorname{Pr} \circ \Xi\left(h_{\sigma}\left(\log \left(\frac{1+\tau}{1-\tau}\right)\right), \hat{v}\left(\log \left(\frac{1+\tau}{1-\tau}\right) ; \Lambda\right)\right) & \text { if } \Lambda=\Lambda_{1}, \Lambda_{2} \text { and } \tau \in(-1,+1) \\ \operatorname{Pr} \circ \Xi(O,[1: 0: 0]) & \text { if } \Lambda \in\left[\Lambda_{1}, \Lambda_{2}\right] \text { and } \tau= \pm 1\end{cases}$

where $\operatorname{Pr}: \bar{\Gamma}_{\sigma} \times S_{0} \rightarrow S_{0}$ is the projection. This $\mathfrak{g}$ induces a homomorphism $\mathfrak{g}_{*}: H_{1}\left(\partial\left(\left[\Lambda_{1}, \Lambda_{2}\right] \times[-1,+1]\right)\right) \rightarrow H_{1}\left(S_{0}\right)$, where both $H_{1}\left(\partial\left(\left[\Lambda_{1}, \Lambda_{2}\right] \times[-1,+1]\right)\right)$ and $H_{1}\left(S_{0}\right)$ are isomorphic to $\mathbb{Z}$. 
Definition 4.1. Let $H_{1}\left(\partial\left(\left[\Lambda_{1}, \Lambda_{2}\right] \times[-1,+1]\right)\right)$ and $H_{1}\left(S_{0}\right)$ be identified with $\mathbb{Z}$. Then the index $I_{\sigma}\left(\Lambda_{1}, \Lambda_{2}\right)$ is defined by

$$
I_{\sigma}\left(\Lambda_{1}, \Lambda_{2}\right):=\left|\mathfrak{g}_{*}(1)\right|
$$

Next proposition shows a basic property of this index.

Proposition 4.3. If $I\left(\Lambda_{1}, \Lambda_{2}\right) \geq N$, there are at least $N$ eigenvalues in the interval $\left[\Lambda_{1}, \Lambda_{2}\right]$.

Proof. This proposition is proven by induction.

First, if $\mathfrak{g}_{*}(1) \neq 0$ then there exists at least one eigenvalue in $\left[\Lambda_{1}, \Lambda_{2}\right]$. In fact, if there is no eigenvalue in the interval, the map $\mathfrak{g}$ can be extended to a continuous map $\overline{\mathfrak{g}}:\left[\lambda_{1}, \lambda_{2}\right] \times[-1,+1] \rightarrow S_{0}$ by lemma 4.3 , and thus it is homotopic to a map which maps whole $\left[\lambda_{1}, \lambda_{2}\right] \times[-1,+1]$ into one point. Therefore $\mathfrak{g}_{*}$ is trivial and $\mathfrak{g}_{*}(1)=0$.

Next, assume that the proposition has been proven up to $N$ and suppose that $\left|\mathfrak{g}_{*}(1)\right| \geq N+1$.

Let $\Lambda^{\prime}$ be the smallest eigenvalue in the interval $\left[\Lambda_{1}, \Lambda_{2}\right]$ and let $\Lambda_{3}$ be a value in $\left[\Lambda^{\prime}, \Lambda_{2}\right]$ so that there is no eigenvalue between $\Lambda^{\prime}$ and $\Lambda_{3}$. Let $\mathfrak{g}_{1}: \partial\left(\left[\Lambda_{1}, \Lambda_{3}\right]\right.$ $\times[-1,+1]) \rightarrow S_{0}, \mathfrak{g}_{2}: \partial\left(\left[\Lambda_{3}, \Lambda_{2}\right] \times[-1,+1]\right) \rightarrow S_{0}$ be similarly defined as $\mathfrak{g}$, then it is easy to see that $\mathfrak{g}_{*}(1)=\mathfrak{g}_{1 *}(1)+\mathfrak{g}_{2 *}(1)$ and $\left|\mathfrak{g}_{1 *}(1)\right| \leq 1$. Thus $\left|\mathfrak{g}_{2 *}(1)\right| \geq N$ and, by applying the assumption, there are at least $N$ eigenvalues in the interval $\left[\Lambda_{3}, \Lambda_{2}\right]$. This means the proposition also holds for $N+1$.

5. Proof of the theorem. The goal of this section is to prove the theorem by calculating the index defined in the previous section. More precisely, let $\tilde{\Lambda}<0$ be a fixed number satisfying $|\tilde{\Lambda}|<\tilde{\Lambda}_{0}$ and set $\Lambda_{1}=\left|\nu_{2}\right|^{\chi} \tilde{\Lambda}$ and let $\Lambda_{2}<0$ be a negative number with small absolute value, then $I_{\sigma}\left(\Lambda_{1}, \Lambda_{2}\right)=N(\sigma)$ shall be proven for small $\left|\nu_{2}\right|$.

5.1. Preliminary analysis. In this part of the section, the flow $\hat{\Phi}^{t}$ is analysed by taking Poincaré maps between $\Sigma_{0} \times \mathbb{R P}^{2}$ and $\Sigma_{1} \times \mathbb{R P}^{2}$. For this purpose, set $T_{n-k+1}^{\prime}$ $(k=1, \cdots, n)$ be the time at which $h_{\sigma}(t)$ hits $C_{\rho}$ for the $k$-th time - notice that $T_{1}^{\prime}=0$ - and let $\Psi: \Sigma_{0} \times \mathbb{R} \mathbb{P}^{2} \rightarrow \Sigma_{1} \times \mathbb{R} \mathbb{P}^{2}$ and $\Psi^{\prime}: \Sigma_{1} \times \mathbb{R} \mathbb{P}^{2} \rightarrow \Sigma_{0} \times \mathbb{R} \mathbb{P}^{2}$ be Poincaré maps defined by the flow $\hat{\Phi}^{t}$. For the later use, two different systems of inhomogeneous coordinates for $\mathbb{R P}^{2}$ are employed, that is, the point $(X, Y, 1,[\xi: \eta: \zeta]) \in \Sigma_{0} \times \mathbb{R P}^{2}$ in homogeneous coordinates is expressed as $(X, Y, 1 ; P, Q)$ with $P:=\frac{\xi}{\zeta}, Q:=\frac{\eta}{\zeta}$ and $(1, y, z,[\xi: \eta: \zeta]) \in \Sigma_{1} \times \mathbb{R}^{2}$ is expressed as $(1, y, z ; q, r)$ with $q:=\frac{\eta}{\xi}, r:=\frac{\zeta}{\xi}$.

First consider $\Psi^{\prime}$. Let $u(t)$ be a solution of (3.1) staying in the cube $\{|x|,|y|,|z| \leq$ $1\}$ for $t_{1} \leq t \leq t_{2}$ which starts in $C_{\rho}$ at $t=t_{1}$ and reaches $R_{\rho}$ at $t=t_{2}$, then straight forward calculation shows the following.

$$
\begin{aligned}
& \text { LEMMA 5.1. If } u\left(t_{1}\right)=(1, y, z) \text { then } u\left(t_{2}\right)=\left(z^{-\frac{\lambda^{s}}{\lambda^{u}}}, y z^{-\frac{\lambda^{u u}}{\lambda^{u}}}, 1\right) \text { and } \\
& \qquad \Psi^{\prime-1}\left(z^{-\frac{\lambda^{s}}{\lambda^{u}}}, y z^{-\frac{\lambda^{u u}}{\lambda^{u}}}, 1 ; P, Q\right)=\left(1, y, z ; \frac{Q}{P} z^{\frac{\lambda^{u u}(\Lambda)-\lambda^{u}(\Lambda)}{\lambda^{s}(0)}}, \frac{1}{P} z^{\frac{\lambda^{u}(\Lambda)-\lambda^{s}(\Lambda)}{\lambda^{u}(0)}}\right)
\end{aligned}
$$

Especially, the tangent vector $u^{\prime}(t)$ along the orbit satisfies

$$
\left(u\left(t_{2}\right) ; u^{\prime}\left(t_{2}\right)\right)=\left(z^{-\frac{\lambda^{s}}{\lambda^{u}}}, y z^{-\frac{\lambda^{u u}}{\lambda^{u}}}, 1 ; \frac{\lambda^{s}}{\lambda^{u}} z^{-\frac{\lambda^{s}}{\lambda^{u}}}, \frac{\lambda^{u u}}{\lambda^{u}} y z^{-\frac{\lambda^{u} u}{\lambda^{u}}}\right)
$$


and

$$
\left(u\left(t_{1}\right) ; u^{\prime}\left(t_{1}\right)\right)=\left(1, y, z ; \frac{\lambda^{u u}}{\lambda^{s}} y, \frac{\lambda^{u}}{\lambda^{s}} z\right)
$$

Next, consider $\Psi$. This time, let $u(t)$ be a solution of (3.1) staying outside the cube $\{|x|,|y|,|z| \leq 1\}$ for $t_{1} \leq t \leq t_{2}$ which starts in $R_{\rho}$ at $t=t_{1}$ and reaches $C_{\rho}$ at $t=t_{2}$. Denote $u\left(t_{1}\right)=(X, Y, 1)$ and $u\left(t_{2}\right)=(1, y, z)$ and let

$$
\Psi^{-1}(1, y, z ; q, r)=\left(X, Y, 1 ; \Psi_{1}(y, z ; q, r), \Psi_{2}(y, z ; q, r)\right)
$$

and expand $\Psi_{1}$ and $\Psi_{2}$ up to first order:

$$
\begin{gathered}
\Psi_{1}(y, z ; q, r)=\Psi_{1}\left(y, z ; \frac{\lambda^{u u}}{\lambda^{s}} y, \frac{\lambda^{u}}{\lambda^{s}} z\right) \\
+\frac{\partial \Psi_{1}}{\partial q}\left(y, z ; \frac{\lambda^{u u}}{\lambda^{s}} y, \frac{\lambda^{u}}{\lambda^{s}} z\right)\left(q-\frac{\lambda^{u u}}{\lambda^{s}} y\right) \\
+\frac{\partial \Psi_{1}}{\partial r}\left(y, z ; \frac{\lambda^{u u}}{\lambda^{s}} y, \frac{\lambda^{u}}{\lambda^{s}} z\right)\left(r-\frac{\lambda^{u}}{\lambda^{s}} z\right) \\
\quad+\left.\frac{\partial \Psi_{1}}{\partial \Lambda}\left(y, z ; \frac{\lambda^{u u}}{\lambda^{s}} y, \frac{\lambda^{u}}{\lambda^{s}} z\right)\right|_{\Lambda=0} \Lambda \\
+(\text { higher order terms }) \\
=\frac{\lambda^{s}}{\lambda^{u}} X+\Psi_{1 q}\left(q-\frac{\lambda^{u u}}{\lambda^{s}} y\right)+\Psi_{1 r}\left(r-\frac{\lambda^{u}}{\lambda^{s}} z\right)+\Psi_{1 \Lambda} \Lambda \\
+ \text { (higher order terms) }
\end{gathered}
$$

and

$$
\begin{aligned}
\Psi_{2}(y, z ; q, r)= & \frac{\lambda^{u u}}{\lambda^{u}} Y+\Psi_{2 q}\left(q-\frac{\lambda^{u u}}{\lambda^{s}} y\right)+\Psi_{2 r}\left(r-\frac{\lambda^{u}}{\lambda^{s}} z\right)+\Psi_{2 \Lambda} \Lambda \\
& + \text { (higher order terms) }
\end{aligned}
$$

Then, for $u(t)=h_{\sigma}(t)$, the following holds.

LEMMA 5.2 .

$$
\Psi_{1 r}=O\left(\left|\nu_{2}\right|\right)
$$

Proof. Let $(X, Y, Z)=\tilde{\Phi}(x, y, z ; t ; \nu)=\left(\tilde{\Phi}_{1}(x, y, z), \tilde{\Phi}_{2}(x, y, z), \tilde{\Phi}_{3}(x, y, z)\right)$ be the time $t$ map defined by the flow induced by (3.1) and let $t=t_{*}(y, z ; \nu)$ be the function satisfying $\tilde{\Phi}\left(1, y, z ; t_{*}(y, z ; \nu) ; \nu\right)=(X, Y, 1)=G_{\nu}^{-1}(1, y, z)$ where $G_{\nu}: \Sigma_{0} \rightarrow$ $\Sigma_{1}$ is the return map defined in Section 2 .

First, the tangent vector $v(t)$ to the orbit $u(t)$ satisfies

$$
\begin{aligned}
v\left(t_{1}\right) & =\left(\lambda^{s} X, \lambda^{u u} Y, \lambda^{u}\right) \\
& =D \tilde{\Phi}\left(1, y, z ; t_{*}(y, z ; \nu) ; \nu\right) v\left(t_{2}\right) \\
v\left(t_{2}\right) & =\left(\lambda^{s}, \lambda^{u u} y, \lambda^{u} z\right)
\end{aligned}
$$


where $u\left(t_{1}\right)=(X, Y, 1)$ and $u\left(t_{2}\right)=(1, x, y)$. This together with $X=O\left(\left|\nu_{2}\right|^{R}\right)$, $y=O\left(\left|\nu_{2}\right|^{\frac{\lambda^{u}}{\lambda^{u}}}\right)$ and $z=O\left(\left|\nu_{2}\right|\right)$ implies that

$$
\frac{\partial \tilde{\Phi}_{1}}{\partial x}=O\left(\left|\nu_{2}\right|\right) \text { and } \frac{\partial \tilde{\Phi}_{3}}{\partial x} \rightarrow \frac{\lambda^{u}}{\lambda^{s}} \text { as } \nu_{2} \rightarrow 0
$$

Second, there exists a constant $c$ so that

$$
c\left|\nu_{2}\right|>\left|\frac{\partial \varphi}{\partial z}(z ; \xi, \nu)\right|
$$

if $\left|\nu_{2}\right|$ is sufficiently small. This is shown in similar manner as (4.1). This means $\frac{\partial X}{\partial z}=O\left(\left|\nu_{2}\right|\right)$ and hence $\frac{\partial \tilde{\Phi}_{1}}{\partial z}=O\left(\left|\nu_{2}\right|\right)$ because $\frac{\partial X}{\partial z}=\frac{\partial \tilde{\Phi}_{1}}{\partial z}-\frac{\lambda^{s}}{\lambda^{u}} X \frac{\partial \tilde{\Phi}_{3}}{\partial z}$ and $\frac{\partial \tilde{\Phi}_{3}}{\partial z}$ is bounded.

On the other hand,

$$
\Psi_{1}(q, r)=\frac{\frac{\partial \tilde{\Phi}_{1}}{\partial x}+\frac{\partial \tilde{\Phi}_{1}}{\partial y} q+\frac{\partial \tilde{\Phi}_{1}}{\partial z} r}{\frac{\partial \tilde{\Phi}_{3}}{\partial x}+\frac{\partial \tilde{\Phi}_{3}}{\partial y} q+\frac{\partial \tilde{\Phi}_{3}}{\partial z} r}
$$

and therefor

$$
\frac{\partial \Psi_{1}}{\partial r}=\frac{\frac{\partial \tilde{\Phi}_{1}}{\partial z}\left(\frac{\partial \tilde{\Phi}_{3}}{\partial x}+\frac{\partial \tilde{\Phi}_{3}}{\partial y} q+\frac{\partial \tilde{\Phi}_{3}}{\partial z} r\right)-\frac{\partial \tilde{\Phi}_{3}}{\partial z}\left(\frac{\partial \tilde{\Phi}_{1}}{\partial x}+\frac{\partial \tilde{\Phi}_{1}}{\partial y} q+\frac{\partial \tilde{\Phi}_{1}}{\partial z} r\right)}{\left(\frac{\partial \tilde{\Phi}_{3}}{\partial x}+\frac{\partial \tilde{\Phi}_{3}}{\partial y} q+\frac{\partial \tilde{\Phi}_{3}}{\partial z} r\right)^{2}}
$$

The lemma immediately follows from this. $\square$

The fact below is also easily seen.

LEMMA 5.3.

$$
-\left.\frac{\lambda^{u}}{\lambda^{s}} \frac{\partial \Psi_{1}}{\partial \Lambda}\left(y, z ; \frac{\lambda^{u u}}{\lambda^{s}} y, \frac{\lambda^{u}}{\lambda^{s}} z\right)\right|_{\Lambda=0} \rightarrow D \quad \text { as } x, y \text { and } \nu_{2} \rightarrow 0
$$

where $D$ is what is defined in the condition ND.

The following three propositions are basis of calculation of the index. For a moment, $D$ is assumed to be negative, but the result for positive $D$ is obtained by simply change the sign of $\Lambda$.

Let $\hat{V}_{i}\left(T_{k} ; \Lambda\right)(i=1,2)$ be defined as

$$
\hat{V}_{1}\left(T_{k} ; \Lambda\right):=\frac{v_{1}\left(T_{k} ; \Lambda\right)}{v_{3}\left(T_{k} ; \Lambda\right)} \quad \hat{V}_{2}\left(T_{k} ; \Lambda\right):=\frac{v_{2}\left(T_{k} ; \Lambda\right)}{v_{3}\left(T_{k} ; \Lambda\right)}
$$

Proposition 5.1. Fix $\tilde{\Lambda}$ satisfying $-\tilde{\Lambda}_{0}<\tilde{\Lambda}<0$, then

$$
\hat{V}_{1}\left(T_{k} ;\left|\nu_{2}\right|^{\chi} \tilde{\Lambda}\right)>0
$$

for sufficiently small $\left|\nu_{2}\right|$.

Proof. Before going into the proof, note that $h_{\sigma 2}\left(T_{k}^{\prime}\right)=O\left(\left|\nu_{2}\right|^{\frac{\lambda^{u u}}{\lambda^{u}}}\right)$ and $h_{\sigma 3}\left(T_{k}^{\prime}\right)=$ $O\left(\left|\nu_{2}\right|\right)$ as $h_{\sigma}\left(T_{k}^{\prime}\right)$ is in ' $C_{\rho} \cap P_{\rho}$ and this implies $h_{\sigma 1}\left(T_{k-1}\right)=O\left(\left|\nu_{2}\right|^{-\frac{\lambda^{s}}{\lambda^{u}}}\right)$, where $h_{\sigma}(T)=\left(h_{\sigma 1}(T), h_{\sigma 2}(T), h_{\sigma 3}(T)\right)$ in $(x, y, z)$-coordinates for $T=T_{k}$ or $T=T_{k}^{\prime}$. 
Then the proposition is proven by induction.

(I) For $k=1$.

$\hat{V}_{1}\left(T_{1} ;\left|\nu_{2}\right|^{\chi} \tilde{\Lambda}\right)>0$ and $\hat{V}_{1}\left(T_{1} ;\left|\nu_{2}\right|^{\chi} \tilde{\Lambda}\right)=O\left(\left|\nu_{2}\right|^{\chi}\right)$ but $\hat{V}_{1}\left(T_{1} ;\left|\nu_{2}\right|^{\chi} \tilde{\Lambda}\right) \neq o\left(\left|\nu_{2}\right|^{\chi}\right)$ follows immediately from the expansion below.

$$
\begin{aligned}
\hat{V}_{1}\left(T_{1} ;\left|\nu_{2}\right|^{\chi} \tilde{\Lambda}\right) & =\Psi(0,0 ; 0,0) \\
& =\frac{\lambda^{s}}{\lambda^{u}} h_{\sigma 1}\left(T_{1}\right)+\Psi_{1 \Lambda}\left|\nu_{2}\right|^{\chi} \tilde{\Lambda}+(\text { higher order terms })
\end{aligned}
$$

(II) For $k \geq 2$. Assume that $\hat{V}_{1}\left(T_{k-1} ;\left|\nu_{2}\right|^{\chi} \tilde{\Lambda}\right)>0$ and $\hat{V}_{1}\left(T_{k-1} ;\left|\nu_{2}\right|^{\chi} \tilde{\Lambda}\right)=O\left(\left|\nu_{2}\right|^{\chi}\right)$ but $\hat{V}_{1}\left(T_{k-1} ;\left|\nu_{2}\right|^{\chi} \tilde{\Lambda}\right) \neq o\left(\left|\nu_{2}\right|^{\chi}\right)$ holds.

Let $\hat{v}_{i}\left(T_{k}^{\prime} ; \Lambda\right)(i=2,3)$ be defined as

$$
\hat{v}_{2}\left(T_{k}^{\prime} ; \Lambda\right):=\frac{v_{2}\left(T_{k}^{\prime} ; \Lambda\right)}{v_{1}\left(T_{k}^{\prime} ; \Lambda\right)} \quad \hat{v}_{3}\left(T_{k}^{\prime} ; \Lambda\right):=\frac{v_{3}\left(T_{k}^{\prime} ; \Lambda\right)}{v_{1}\left(T_{k}^{\prime} ; \Lambda\right)}
$$

Then, by lemma $5.1, \hat{v}_{3}\left(T_{k}^{\prime} ;\left|\nu_{2}\right|^{\chi} \tilde{\Lambda}\right)$ is expressed as

$$
\hat{v}_{3}\left(T_{k}^{\prime} ;\left|\nu_{2}\right|^{\chi} \tilde{\Lambda}\right)=\frac{1}{\hat{V}_{1}\left(T_{k-1} ;\left|\nu_{2}\right| \chi \tilde{\Lambda}\right)} h_{\sigma 3}\left(T_{k}^{\prime}\right)^{\frac{\lambda^{u}(\Lambda)-\lambda^{s}(\Lambda)}{\lambda^{u}(0)}}
$$

and thus $\hat{v}_{3}\left(T_{k}^{\prime} ;\left|\nu_{2}\right|^{\chi} \tilde{\Lambda}\right)=o\left(\left|\nu_{2}\right|\right)$. Moreover, this fact and lemma 4.1 implies $\hat{v}_{2}\left(T_{\prime}^{\prime}:\left|\nu_{0}\right| \chi \tilde{\Lambda}\right)=o\left(\left|\nu_{2}\right|^{\chi}\right)$. This leads to the following estimate:

$$
\begin{aligned}
1 & \left.\hat{\lambda^{u}} ;\left|\nu_{2}\right|^{\chi} \tilde{\Lambda}\right)= \\
& +\Psi_{1 r}\left(\hat{v}_{3}\left(T_{k}^{\prime} ;\left|\nu_{2}\right|^{\chi} \tilde{\Lambda}\right)-\frac{\lambda^{u}}{\lambda^{s}} h_{\sigma 3}\left(T_{k}^{\prime}\right)\right)+\Psi_{1 \Lambda}\left|\nu_{2}\right|^{\chi} \tilde{\Lambda} \\
& \quad+(\text { higher order terms) }
\end{aligned}
$$

Then, $\hat{V}_{1}\left(T_{k} ;\left|\nu_{2}\right|^{\chi} \tilde{\Lambda}\right)>0$ and $\hat{V}_{1}\left(T_{k} ;\left|\nu_{2}\right|^{\chi} \tilde{\Lambda}\right)=O\left(\left|\nu_{2}\right|^{\chi}\right)$ but $\hat{V}_{1}\left(T_{k} ;\left|\nu_{2}\right|^{\chi} \tilde{\Lambda}\right) \neq$ $o\left(\left|\nu_{2}\right|^{\chi}\right)$ immediately follows. $\square$

On the other hand the $\hat{V}_{1}\left(T_{k} ; \Lambda\right)$ has the opposite sign for small $\Lambda$.

Proposition 5.2. If $\Lambda<0$ and $|\Lambda|$ is sufficiently small, then

$$
\hat{V}_{1}\left(T_{k} ; \Lambda\right)<0
$$

holds for $k=1, \cdots, n-1$

Proof. This is easily seen from the fact that

$$
\hat{V}_{1}\left(T_{k} ; 0\right)=\frac{\lambda^{s}}{\lambda^{u}} h_{\sigma 1}\left(T_{k}\right)<0
$$

The other proposition concerns changes of the orientation of $\hat{\mathcal{C}}$ along the flow.

Proposition 5.3. Let $i_{k}: \hat{\mathcal{C}} \hookrightarrow\left\{h_{\sigma}\left(T_{k}\right)\right\} \times \hat{\mathcal{C}} \subset \bar{\Gamma}_{0} \times \mathbb{R P}^{2}$ be the inclusion and $P: \bar{\Gamma}_{0} \times \mathbb{R P}^{2} \rightarrow \mathbb{R P}^{2}$ be the projection, and define

$$
\Theta_{k}:=P \circ \hat{\Phi}^{T_{k}-T_{k-1}} \circ i_{k-1}: \hat{\mathcal{C}} \rightarrow \hat{\mathcal{C}}
$$


for $k=2, \cdots, n$.

Then $\Theta_{k *}: H_{1}(\hat{\mathcal{C}}) \rightarrow H_{1}(\hat{\mathcal{C}})$ is equal to id: $H_{1}(\hat{\mathcal{C}}) \rightarrow H_{1}(\hat{\mathcal{C}})$ if $\sigma_{k-1}=+1$ and - id: $H_{1}(\hat{\mathcal{C}}) \rightarrow H_{1}(\hat{\mathcal{C}})$ if $\sigma_{k-1}=-1$.

Proof. First, as stated in [9], $g_{Y}^{1}(0,0 ; 0)=0$ and $g_{X}^{1}(0,0 ; 0) g_{Y}^{2}(0,0 ; 0)<0$.

If $g_{X}^{1}(0,0 ; 0) g_{Y Y}^{1}(0,0 ; 0)>0$ (inward twist case), the assumption that $g_{Y Y}^{1}(0,0 ; 0)$ $>0$ means $g_{X}^{1}(0,0 ; 0)>0$ and $g_{Y}^{2}(0,0 ; 0)<0$. Thus $\nu_{2}<0$ is equal to $g_{Y}^{1}\left(0, Y_{*}(\nu) ; \nu\right)$ $>0$. This implies $g_{Y}^{1}\left(h_{\sigma}\left(T_{k}\right) ; \nu\right)>0$ if $h_{\sigma}\left(T_{k}^{\prime}\right)=\left(g^{1}\left(h_{\sigma}\left(T_{k}\right) ; \nu\right), g^{2}\left(h_{\sigma}\left(T_{k}\right) ; \nu\right)\right)$ is in the component of $C_{\rho} \cap P_{\rho}$ which includes $(y, z)=(0,0)\left(i . e . \sigma_{k}=+1\right)$, and $g_{Y}^{1}\left(h_{\sigma}\left(T_{k}\right) ; \nu\right)<0$ if $h_{\sigma}\left(T_{k}^{\prime}\right)$ is in the other component (i.e. $\left.\sigma_{k}=-1\right)$.

On the other hand, if $g_{X}^{1}(0,0 ; 0) g_{Y Y}^{1}(0,0 ; 0)<0$ (outward twist case), $g_{X}^{1}(0,0 ; 0)$ $<0$ and $g_{Y}^{2}(0,0 ; 0)>0$. Thus $\nu_{2}<0$ is equal to $g_{Y}^{1}\left(0, Y_{*}(\nu) ; \nu\right)<0$. This implies $g_{Y}^{1}\left(h_{\sigma}\left(T_{k}\right) ; \nu\right)<0$ if $h_{\sigma}\left(T_{k}^{\prime}\right)$ is in the component of $C_{\rho} \cap P_{\rho}$ which includes $(y, z)=(0,0)$ (i.e. $\sigma_{k}=-1$ ), and $g_{Y}^{1}\left(h_{\sigma}\left(T_{k}\right) ; \nu\right)>0$ if $h_{\sigma}\left(T_{k}^{\prime}\right)$ is in the other component (i.e. $\left.\sigma_{k}=+1\right)$.

Therefore, if $\sigma_{k}=+1$, then the half-line $\left\{h_{\sigma}\left(T_{k}\right)\right\} \times\left\{\left(0, V_{2}, 0\right) \mid V_{2}>0\right\}$ is mapped into $\left\{h_{\sigma}\left(T_{k}^{\prime}\right)\right\} \times\left\{\left(v_{1}, v_{2}, v_{3}\right)|| \nu_{2}\left|\chi \sqrt{\left|v_{1}\right|^{2}+\left|v_{3}\right|^{2}}<c^{\prime}\right| v_{2} \mid, v_{2}>0\right\}$ by $\Phi^{T_{k}}-T_{k}^{\prime}$, and if $\sigma_{k}=-1$, then into $\left\{h_{\sigma}\left(T_{k}^{\prime}\right)\right\} \times\left\{\left.\left(v_{1}, v_{2}, v_{3}\right)|| \nu_{2}\right|^{\chi} \sqrt{\left|v_{1}\right|^{2}+\left|v_{3}\right|^{2}}<c^{\prime}\left|v_{2}\right|, v_{2}<0\right\}$. The proposition follows from this and the fact that $\Phi^{T_{k}-T_{k}^{\prime}}$ is orientation preserving.

5.2. Proof of the theorem. In this section, the index is calculated and the theorem is proven.

Let $\Omega_{1}, \Omega_{2}$ be some small neighborhood of $[0: 0: 1]$ and $[1: 0: 0]$ of $\mathbb{R P}^{2}$ and $\iota_{1}, \iota_{2}:[0,+1] \rightarrow \mathbb{R P}^{2}$ be defined as

$$
\iota_{1}(\xi)=[t: 0: 1-t], \iota_{2}(\xi)=[-t: 0: 1-t]
$$

and let $\Omega_{i k}:=\operatorname{Pr} \circ \Xi\left(h_{\sigma}\left(T_{k}\right), \Omega_{i}\right) \subset S_{0}$ and $\iota_{i k}:=\operatorname{Pr} \circ \Xi\left(h_{\sigma}\left(T_{k}\right), \iota_{i}(\cdot)\right):[0,1] \rightarrow S_{0}$. Here is a homology exact sequence

$$
\begin{aligned}
\cdots \rightarrow & \tilde{H}_{1}\left(\bigcup_{k}\left(\Omega_{1 k} \cup \Omega_{2 k}\right)\right) \stackrel{i_{*}}{\rightarrow} \tilde{H}_{1}\left(S_{0}\right) \\
& \stackrel{j_{*}}{\rightarrow} H_{1}\left(S_{0}, \bigcup_{k}\left(\Omega_{1 k} \cup \Omega_{2 k}\right)\right) \stackrel{\partial_{*}}{\rightarrow} \tilde{H}_{0}\left(\bigcup_{k}\left(\Omega_{1 k} \cup \Omega_{2 k}\right)\right) \rightarrow \cdots
\end{aligned}
$$

where $\tilde{H}_{*}$ stands for reduced homology group and $\tilde{H}_{1}\left(S_{0}\right)$ can be identified with $H_{1}\left(S_{0}\right)$. Moreover $j_{*}$ is injective and $j_{*}\left(H_{1}\left(S_{0}\right)\right)$ is generated by $\left(\left[\iota_{1 k}\right]-\left[\iota_{2 k}\right]\right)$ for arbitrary $k$.

Choose a generator $1 \in H_{1}\left(S_{0}\right) \cong \mathbb{Z}$ so that $j_{*}(1)=\left[\iota_{11}\right]-\left[\iota_{21}\right]$ Then, proposition 5.3 implies the following.

Proposition 5.4. $j_{*}(1)=\left[\iota_{1 k}\right]-\left[\iota_{2 k}\right]$ if the product $\sigma_{1} \cdots \sigma_{k-1}=+1$ and $j_{*}(1)=-\left[\iota_{1 k}\right]+\left[\iota_{2 k}\right]$ if $\sigma_{1} \cdots \sigma_{k-1}=-1$

Proof. This proposition easily follows from the fact that the diagram below com- 
mutes.

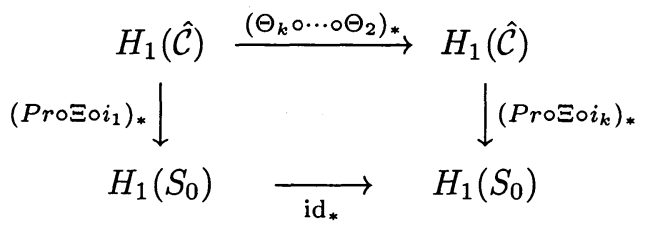

On the other hand, proposition 5.1 and 5.2 implies next.

Proposition 5.5. For $k=1, \cdots, n-1, \operatorname{Pr} \circ \Xi\left(\hat{v}\left(T_{k}+\left(T_{k+1}^{\prime}-T_{k}\right) t ;\left|\nu_{2}\right|^{\chi} \tilde{\Lambda}\right)\right)$ is homotopic to $\iota_{1 k+1}(t)$ as a map $([0,1],\{0,1\}) \rightarrow\left(S_{0}, \bigcup_{k}\left(\Omega_{1 k} \cup \Omega_{2 k}\right)\right)$, whereas $\operatorname{Pr} \circ \Xi\left(\hat{v}\left(T_{k}+\left(T_{k+1}^{\prime}-T_{k}\right) t ; \Lambda\right)\right)$ is homotopic to $\iota_{2 k+1}(t)$ when $\Lambda<0$ and $|\Lambda|$ is sufficiently small.

For $k=n, \operatorname{Pr} \circ \Xi\left(\hat{v}\left(T_{n}+\left(T-T_{n}\right) t ;\left|\nu_{2}\right|^{\chi} \tilde{\Lambda}\right)\right)$ is also homotopic to $\iota_{1 n}(t)$ whereas $\operatorname{Pr} \circ \Xi\left(\hat{v}\left(T_{n}+\left(T-T_{n}\right) t ; \Lambda\right)\right)$ for $T$ large enough can be both $\iota_{1 n}(t)$ or $\iota_{2 n}(t)$.

$\operatorname{Pr} \circ \Xi\left(\hat{v}\left(T_{n}+\left(T-T_{n}\right) t ;\right)\right)$ can be determined by standard argument concerning what is called Evans' function.(See [21].) Only results presented here without proof.

Lemma 5.4. $\operatorname{Pr} \circ \Xi\left(\hat{v}\left(T_{n}+\left(T-T_{n}\right) t ; \Lambda\right)\right)$ is homotopic to $\iota_{1 n}(t)$ if $\sigma_{1} \cdots \sigma_{n-1}=$ +1 and homotopic to $\iota_{2 n}(t)$ if $\sigma_{1} \cdots \sigma_{n-1}=-1$

Finally the theorem is proven.

Proof. (Proof of the theorem) First, notice that

$$
\mathfrak{g}_{*}: H_{1}\left(\partial\left(\left[\left|\nu_{2}\right|^{\chi} \tilde{\Lambda}, \Lambda\right] \times[-1,+1]\right)\right) \rightarrow H_{1}\left(S_{0}, \bigcup_{k}\left(\Omega_{1 k} \cup \Omega_{2 k}\right)\right)
$$

coincides with the composition of $\mathfrak{g}_{*}: H_{1}\left(\partial\left(\left[\left|\nu_{2}\right| \chi \tilde{\Lambda}, \Lambda\right] \times[-1,+1]\right)\right) \rightarrow H_{1}\left(S_{0}\right)$ and $j_{*}$. Then, from proposition 5.5 and lemma 5.4,

$$
\mathfrak{g}_{*}(1)=\sum_{k=1}^{n-1}\left[\iota_{1 k}\right]-\left[\iota_{2 k}\right]+\left\{\begin{array}{ccc}
{\left[\iota_{1 n}\right]-\left[\iota_{2 n}\right]} & \text { if } \sigma_{1} \cdots \sigma_{n-1}=-1 \\
0 & \text { if } & \sigma_{1} \cdots \sigma_{n-1}=+1
\end{array}\right.
$$

in $H_{1}\left(S_{0}, \bigcup_{k}\left(\Omega_{1 k} \cup \Omega_{2 k}\right)\right)$. Therefore by proposition 5.4 ,

$$
I_{\sigma}\left(\left|\nu_{2}\right|^{\chi} \tilde{\Lambda}, \Lambda\right)=\mid 1+\sum_{k=1}^{n-2} \sigma_{1} \cdot \sigma_{2} \cdots \sigma_{k}+\left\{\begin{array}{ccc}
-1 & \text { if } & \sigma_{1} \cdots \sigma_{n-1}=-1 \\
0 & \text { if } & \sigma_{1} \cdots \sigma_{n-1}=+1
\end{array} \mid\right.
$$

and thus the theorem holds.

Proof. (Proof of corollary to the theorem) First, $D$ has to be negative for the original pulse to be stable. This is because

$$
\operatorname{sign}\left\{\operatorname{det}\left(v_{0}\left(t_{0}, \Lambda\right),(0,1,0),(0,0,1)\right)\right\}
$$

for large positive $\Lambda$ coincides with that for small positive $\Lambda$ if there is no eigenvalue between them. On the other hands, it coincides with

$$
\operatorname{sign}\{\operatorname{det}((-1,0,0),(0,1,0),(0,0,1))\}
$$


by standard argument of Evans' function. (See [21]). The conclusion is obtained immediately from this.

Second, under the assumption, there are $n$ critical eigenvalues near origin and other eigenvalues have negative real part.(See [2] or [11] for this fact.)

Therefore applying the theorem, there are $n-1$ negative eigenvalues near origin and the other one is at origin. Thus the $n$-pulse is stable.

Acknowledgment. The author expresses his sincere gratitude to C.Jones for support and encouragement, and to the kind hospitality of Brown University. He is also grateful to B.Sandstede for stimulating discussions. This work is supported by NFS grant DMS-970496

6. Appendix. This section is devoted to brief summary of topological notions. For more details, consult some textbooks on algebraic topology such as [19].

6.1. The projective space. First, definition of the projective space is introduced.

Let $\sim$ be an equivalent relation on $\mathbb{R}^{n+1} \backslash\{0\}$ defined as follows:

$x \sim y$ if and only if there exists a constant $c \in \mathbb{R} \backslash\{0\}$ such that $x=c y$.

Then the projective space is defined below.

Definition 6.1. The quotient space $\mathbb{R P}^{n}:=\left(\mathbb{R}^{n+1} \backslash\{0\}\right) / \sim$ with the quotient topology is called the $n$-dimensional (real)projective space.

This space has a structure of a manifold and a coordinate system can be defined in the following manner.

Let $x=\left(x_{0}, x_{1}, \ldots, x_{n}\right)$ be a point in $\mathbb{R}^{n+1} \backslash\{0\}$ then the ratios $x_{0}: x_{1}: \cdots: x_{n}$ defines a point in $\mathbb{R P}^{n}$ therefore the equivalent class of $[x] \in \mathbb{R} \mathbb{P}^{n}$ is written as $\left[x_{0}\right.$ : $\left.x_{1}: \cdots: x_{n}\right]$. Moreover, if $x_{i} \neq 0$ then this expression can be normalized so that $[x]=$ $\left[\frac{x_{0}}{x_{i}}: \frac{x_{1}}{x_{i}}: \cdots: \stackrel{i \text {-th }}{1}: \cdots: \frac{x_{n}}{x_{i}}\right]$, thus there is a homeomorphism $\varphi_{i}$ from $U_{i}:=\left\{\left[x_{0}: \cdots:\right.\right.$ $\left.\left.x_{n}\right] \mid x_{i} \neq 0\right\} \subset \mathbb{R P}^{n}$ to $\mathbb{R}^{n}$ such that $\varphi_{i}\left(\left[x_{0}: \cdots: x_{n}\right]\right)=\left(\frac{x_{0}}{x_{i}}, \ldots, \frac{x_{i-1}}{x_{i}}, \frac{x_{i+1}}{x_{i}}, \ldots, \frac{x_{n}}{x_{i}}\right)$. The pair $\left(\varphi_{i}, U_{i}\right)$ is employed as a local chart and $\Phi=\left\{\left(\varphi_{i}, U_{i}\right)\right\}_{i=1}^{n}$ becomes an atlas. Next, let

$$
\dot{x}=A(t) x
$$

be a linear ordinary differential equation on $\mathbb{R}^{n+1}$, and let $x_{1}(t)$ and $x_{2}(t)$ be two non-trivial solutions of this equation. If $x_{1}\left(t_{0}\right)$ and $x_{2}\left(t_{0}\right)$ belong to same equivalence class of $\mathbb{R P}^{n}$ i.e. $\left[x_{1}\left(t_{0}\right)\right]=\left[x_{2}\left(t_{0}\right)\right]$, then $\left[x_{1}(t)\right]=\left[x_{2}(t)\right]$ holds for all $t$ because of linear nature of the equation. This is the way in which the equation induces a flow on $\mathbb{R P}^{n}$.

Assume that $A(t)=A$ is independent of $t$, and let $\mathbf{e}$ be an eigenvector with eigenvlue $\lambda$ and assuem that any other eigenvalue has the real part which is smaller than $\operatorname{Re} \lambda$. Then $[v]$ is an equilibrium of the induced flow because $\operatorname{span}\{v\}$ is a invariant subspace of (6.1). Moreover, $[v]$ is an attracting equilibria, because any other solution nearby approaches to this subspace as time evolves. Similarly, an eigenspace for an eigenvalue whith smallest real part corresponds to a repelling equilibria. Similar argument holds true if $A(t) \rightarrow A$ as $t \rightarrow \pm \infty$.

6.2. The homology group. First, the homology group is defined.

The following set is called the $n$-simplex.

$$
\Delta^{n}:=\left\{x=\left(x_{0}, x_{1}, \ldots, x_{n}\right) \in \mathbb{R}^{n+1} \mid 0 \leq x_{i}, \sum_{i=0}^{n} x_{i}=1\right\}
$$


$\Delta^{n-1}$ can be identified with $\Delta_{j}^{n-1}:=\left\{x=\left(x_{0}, x_{1}, \ldots, x_{n}\right) \in \Delta^{n} \mid x_{j}=0\right\}$ and the inclusion map $\Delta_{j}^{n-1} \hookrightarrow \Delta^{n}$ is denoted by $\varepsilon_{j}^{n}$ for each $j$.

Let $X$ be a topological space then a continuous map $\sigma: \Delta^{n} \rightarrow X$ is called singular $n$-simplex. The free module generated by the set of all singular $n$-simples is denoted by $S_{n}(X)$, then $S_{n}(X)$ is defined to be $\{0\}$ for $n<0$. Let $\partial_{j}(\sigma):=\sigma \circ \varepsilon_{j}^{n}$ and let the boundary homomorphism $\partial: S_{n}(X) \rightarrow S_{n-1}(X)$ be defined by

$$
\partial=\sum_{j=0}^{n}(-1)^{j} \partial_{j} \quad(n>0), \quad \partial=0 \quad(n \leq 0)
$$

Definition 6.2. The $n$-dimensional homology group of the topological space $X$ is defined by

$$
H_{n}(X):=\operatorname{ker} \partial / \operatorname{Im} \partial
$$

where ker $\partial$ is the kernel of the map $\partial: S_{n}(X) \rightarrow S_{n-1}(X)$ and $\operatorname{Im} \partial$ is the image of the map $\partial: S_{n+1}(X) \rightarrow S_{n}(X)$.

If $\tilde{\Delta}^{n}$ is homeomorphic to $\Delta^{n}$ and $\phi: \Delta^{n} \rightarrow \tilde{\Delta}^{n}$ is a homeomorphism, then a continuous map $\tilde{\sigma}: \tilde{\Delta}^{n} \rightarrow X$ can be regarded as an $n$-simplex by identifying it with $\tilde{\sigma} \circ \phi$. A choice of such a $\phi$ determines the sign of $[\tilde{\sigma}] \in H_{n}(X)$.

If $Y$ is a subspace of $X$, then $S(Y)$ is a submodule of $S(X)$ and $\partial$ induces a homomorphism from $S_{n}(X, Y):=S_{n}(X) / S_{n}(Y)$ to $S_{n-1}(X, Y):=S_{n-1}(X) / S_{n-1}(Y)$. This homomorphism is also denoted by $\partial$.

Definition 6.3. The n-dimensional homology group of the pair of topological spaces $(X, Y)$ is defined by

$$
H_{n}(X, Y):=\operatorname{ker} \partial / \operatorname{Im} \partial
$$

where ker $\partial$ is the kernel of the map $\partial: S_{n}(X, Y) \rightarrow S_{n-1}(X, Y)$ and $\operatorname{Im} \partial$ is the image of the map $\partial: S_{n+1}(X, Y) \rightarrow S_{n}(X, Y)$.

When $X$ is not empty, a homomorphism $\varepsilon: S_{0}(X) \rightarrow \mathbb{Z}$ is defined by $\varepsilon\left(\sum a_{x} x\right)=$ $\sum a_{x},(x \in X)$. Let $\tilde{S}_{n}(X)=S_{n}(X)(n \geq 0) \tilde{S}_{-1}(X)=\mathbb{Z}$ and $\tilde{S}_{n}(X)=0(n<0)$, and let $\partial=\varepsilon$ for $\tilde{S}_{0}(X) \rightarrow \tilde{S}_{-1}(X)$.

DEFINITION 6.4. The $n$-dimensional reduced homology group of the topological space $X$ is defined by

$$
\tilde{H}_{n}(X):=\operatorname{ker} \partial / \operatorname{Im} \partial
$$

where $\operatorname{ker} \partial$ is the kernel of the map $\partial: \tilde{S}_{n}(X) \rightarrow \tilde{S}_{n-1}(X)$ and Im $\partial$ is the image of the map $\partial: \tilde{S}_{n+1}(X) \rightarrow \tilde{S}_{n}(X)$.

Next, a continuous map induces a homomorphism between homology groups.

Let $X$ and $Y$ be topological spaces and $f: X \rightarrow Y$ be a continuous map. Then $f_{\sharp}: S_{n}(X) \rightarrow S_{n}(Y)$ is defined by $f_{\sharp}(\sigma):=f \circ \sigma$.

DeFinition 6.5. A homomorphism between homology groups $f_{*}: H_{n}(X) \rightarrow$ $H_{n}(Y)$ is the induced homomorphism by $f_{\sharp}$.

The important property of the homology group is as follows.

Let $\theta: \Delta^{1} \rightarrow \mathbb{S}^{1}$ be such that $\theta(x, 1-x):=e^{i x}$, then $[\theta] \in H_{1}\left(\mathbb{S}^{1}\right)$ is the generator of $H_{1}\left(\mathbb{S}^{1}\right) \cong \mathbb{Z}$. Moreover, $\left[\theta_{k}\right]=k$ in $H_{1}\left(\mathbb{S}^{1}\right)$ for $\theta_{k}(x, 1-x):=e^{i k x}$.

Therefore, if $f: \mathbb{S}^{1} \rightarrow \mathbb{S}^{1}$ is a continuous map such that $f_{*}(1)=k$, then $f$ maps $\mathbb{S}^{1}$ onto $k$-fold of $\mathbb{S}^{1}$. Same holds true if $X$ is homotopic to $\mathbb{S}^{1}$. 


\section{REFERENCES}

[1] J. Alexander, R. GARDNer, AND C. Jones, A topological invariant arising in the stability analysis of travelling waves, J. Reine Angew. Math., 410 (1990), pp. 167-212.

[2] J. Alexander AND C. Jones, Existence and stability of asymptotically oscillatory double pulses, J. Reine Angew. Math., 446 (1994), pp. 49-79.

[3] — Existence and stability of asymptotically oscillatory triple pulses, Z. Angew. Math. Phys., 44 (1993), pp. 189-200.

[4] J. Evans, Nerve axon equations, I: Linear approximations, Indiana Univ. Math. J., 21 (1972), pp. 877-885.

[5] - Nerve axon equations, II: Stability at rest, Indiana Univ. Math. J., 22 (1972), pp. 75-90.

[6] - Nerve axon equations, III: Stability of the nerve impulse, Indiana Univ. Math. J., 22(1972), pp. 577-594.

[7] J. Evans, N. Fenichel, AND A. Feroe, Double impulse solutions in nerve axon equations, SIAM J. Appl. Math., 42 (1982), pp. 219-234.

[8] D. Henry, The Geometric Theory of Semilinear Parabolic Equations, Lec. Notes in Math. 840, Springer, 1981.

[9] A. Homburg, H. Kokubu, AND M. KRUPA, The cusp horseshoe and its bifurcations in the unfolding of an inclination-flip homoclinic orbit, Erg. The. Dyn. Sys., 14 (1994), pp. 667693.

[10] A. Homburg AND M. KRAUSKOPF, Resonant homoclinic flip bifurcations, preprint.

[11] S. NII, An extension of the stability index for travelling wave solutions and its application for bifurcations, SIAM J. Math. Anal., 28(1997), pp. 402-433.

[12] - Stability of travelling multiple-front (multiple-back) wave solutions of the FitzHughNagumo equations, SIAM J. Math. Anal., 28 (1997), pp. 1094-1112.

[13] - A topological proof of stability of $N$-front solutions of the FitzHugh-Nagumo equations, J. Dyn. Diff. Eqns., 11 (1999), pp. 515-555.

[14] S. NiI AND B. SANDSTEDE, On the connection between the geometry and stability of multi-bump pulses arising in homoclinic fip bifurcations, in preparation.

[15] B. SANDSTEDE, Verzweigungstheorie homokliner Verdopplungen, Ph.D. thesis, University of Stuttgart, 1993.

[16] - Stability of multiple-pulse solutions, Trans. Amer. Math. Soc., 350 (1998), pp. 429-472.

[17] Stability of $N$-fronts bifurcating from a twisted heteroclinic loop and an application to the FitzHugh-Nagumo equation, SIAM J. Math. Anal., 29 (1998), pp. 183-207.

[18] B. SANDSTEDE, J. AlEXANDER, AND C. JONES, Existence and stability of n-pulses on optical fibers with phase-sensitive amplifiers, Physica D, 106 (1997), pp. 167-206.

[19] E. SPANIER, Algebraic Topology, McGraw-Hill series in higher mathematics, McGraw-Hill, 1996.

[20] E. YANAGIDA, Branching of double-pulse solutions from single pulse solutions in nerve axon equations, J. Diff. Eqs., 66 (1987), pp. 243-262.

[21] E. YANAGIDA AND K. MAGINU, Stability of double-pulse solutions in nerve axon equations, SIAM J. Appl. Math., 49 (1989), pp. 1158-1173.

[22] A. YEW, Analytical study of solitary-waves in quadratic media, Ph.D. thesis, Brown University, 1998. 
